Acta Zoológica Mexicana (nueva serie), Volumen 37, 1-25.

https://doi.org/10.21829/azm.2021.3712306

Artículo original

\title{
Arañas edáficas del clado RTA (Arachnida: Araneae) del sur de Tonatico, Estado de México, México
}

\section{Ground-Spiders of RTA clade (Arachnida: Araneae) of southern Tonatico, Estado de México, Mexico}

\section{(iD) 1 DARIANA R. GUERRERO-FUENTES, (iD 2* MARCO ANTONIO DESALES-LARA}

${ }^{1}$ Colección Nacional de Arácnidos (CNAN), Instituto de Biología, Universidad Nacional Autónoma de México (UNAM), Av. Universidad. No. Exterior 3000. Col. UNAM, CU. Delegación Coyoacán. Ciudad de México, México. C.P. 04510.

${ }^{2}$ Facultad de Ciencias Agrícolas, Universidad Autónoma del Estado de México (UAEMéx), Campus El Cerrillo. Carretera Toluca-Ixtlahuaca Kilómetro 15.5, El Cerrillo, Piedras Blancas, C.P. 50200, Toluca, Estado de México, México.

Editor responsable: María Luisa Jiménez-Jiménez

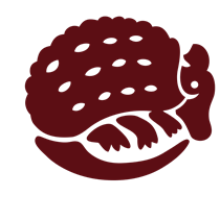

OPEN ACCESS

*Autor corresponsal:

Marco Antonio Desales-Lara madesales@gmail.com

Cita:

Guerrero-Fuentes, D. R., DesalesLara, M. A. (2021) Arañas edáficas del clado RTA (Arachnida: Araneae) del sur de Tonatico, Estado de México, México. Acta Zoológica Mexicana (nueva serie), 37, 1-25.

10.21829/azm.2021.3712306 elocation-id: e3712306

Recibido: 01 julio 2020 Aceptado: 11 marzo 2021

Publicado: 17 junio 2021
RESUMEN. Los estudios ecológicos en México sobre arañas del clado RTA son casi nulos. Por lo tanto, el presente trabajo se enfocó en analizar las variaciones en la abundancia, riqueza, diversidad, composición y estructura de las comunidades de arañas del clado RTA entre las temporadas de lluvias y secas, en tres sitios con diferente tipo de vegetación. Los sitios de muestreo fueron un pastizal inducido, un bosque de pino y una selva baja caducifolia en Tonatico, Estado de México. Las arañas fueron recolectadas mensualmente de noviembre del 2011 a octubre del 2012, usando trampas de caída en cada sitio. Se recolectaron 805 arañas, agrupadas en 16 familias, 29 géneros y 49 especies. Las familias mejor representadas fueron Lycosidae, Gnaphosidae, Salticidae y Corinnidae; mientras que Pardosa medialis, $P$. pauxilla y $P$. marialuisae fueron las especies más abundantes. Aunque no encontramos diferencias estadísticas en la abundancia, riqueza y diversidad entre los diferentes tipos de vegetación, la composición de especies entre sitios si fue estadísticamente diferente. También encontramos que en los tres sitios en la temporada de lluvias hubo mayor cantidad de 
organismos y mayor número de especies. Además, las especies compartidas entre los tres sitios presentaron diferente categoría ecológica. Las diferencias en cuanto a la composición y estructura de las comunidades de arañas las atribuimos a la disimilitud en el tipo de vegetación de cada sitio. Asimismo, inferimos que en la temporada de lluvias hay mayor abundancia y riqueza de arañas debido a que hay mayor disponibilidad de recursos. Para estudios futuros sugerimos evaluar variables microclimáticas para entender mejor la composición y estructura de las comunidades de arañas del clado RTA.

Palabras clave: ensambles ecológicos; arañas epigeas; estacionalidad; inventarios biológicos

ABSTRACT. Ecological studies about spiders within the RTA clade are scarce in Mexico. In the present we analyze the abundance, species richness, diversity, composition, and structure of RTAspider communities in three sites with different vegetation and between rainy and dry season. The sites correspond to human-induced grassland, pine forest and deciduous tropical forest of southern Tonatico, Estado de México. Spiders were collected monthly, from November 2011 to October 2012, using pitfall-traps at each site. We collected 805 spiders from 16 families, 29 genera and 49 species. The most abundant families were Lycosidae, Gnaphosidae, Salticidae and Corinnidae; while the most abundant species were Pardosa medialis, P. pauxilla and P. marialuisae. We did not find differences in the abundance, richness, or diversity between sites. However, we found differences in the species composition at each site and a general pattern of high abundance and species richness in the rainy season. Our results showed a distinct ecological category of shared species between sites. We suggested that differences in structure and composition of spider communities are a response to dissimilarities in vegetation, and the seasonal pattern is related with the availability of resources of the rainy season. Future investigations should evaluate microclimatic variables to understand, on a better way, the composition and structure of RTAspider communities.

Key words: ecological assembles; epigean spiders; seasonality; biological inventories

\section{INTRODUCCIÓN}

Dentro de los arácnidos (excluyendo a los ácaros), el orden Araneae destaca por ocupar el primer lugar en diversidad de especies (Roskov et al., 2019). El clado RTA agrupa 40 familias, 2,078 géneros y 24,753 especies de las cuales alrededor de 29 familias y 1,111 especies se distribuyen en México (Platnick, 2020; WSC, 2020). Este clado de arañas se define por la presencia de una apófisis retro-lateral en la tibia (RTA por sus siglas en inglés) del pedipalpo del macho (Griswold \& Ramírez, 2017), además del incremento de tricobótrias en los segmentos de las patas; esta última característica se correlaciona con una dependencia menor de la telaraña para la captura de presas (Ramírez, 2014; Garrison et al., 2016; Wheeler et al., 2016), pues la detección de vibraciones mecánicas les permite emboscar, acechar o perseguir a sus presas, rasgo que las diferencia de las arañas tejedoras (Foelix, 2011).

Las arañas en general son un buen modelo para realizar estudios ecológicos, ya que son depredadores abundantes dentro de las redes tróficas, su taxonomía es relativamente bien 
conocida y la estructura de sus comunidades y abundancia se correlaciona con atributos del hábitat (Campuzano et al., 2016). Factores físicos como la temperatura, la humedad, el viento, y biológicos como la disponibilidad de alimentos, el tipo de vegetación y de presas, la presencia de especies competidoras y de enemigos naturales limitan los ambientes donde las arañas pueden establecerse (Blanco-Vargas et al., 2003; Corcuera et al., 2008; Corcuera et al., 2010; Foelix, 2011). Además, por ser de los principales depredadores de insectos y por su capacidad para colonizar diferentes ecosistemas, las arañas han sido consideradas de utilidad para la conservación y control biológico de diferentes agroecosistemas (Ibarra-Núñez, 1990; Ibarra-Núñez \& García-Ballinas, 1998).

En el último lustro en México se han realizado diversos estudios que abordan diferentes aspectos ecológicos de la araneofauna, entre ellos están los realizados en el bosque mesófilo de montaña (Campuzano et al., 2016; Campuzano et al., 2019; Campuzano et al., 2020) y los cafetales de Chiapas (Marín et al., 2016), en los cultivos de cacao en Chiapas (Lucio-Palacio \& Ibarra Núñez, 2015) y Tabasco (Cruz-Pérez et al., 2015), en zonas áridas de Coahuila (Bizuet-Flores et al., 2015) y Chihuahua (Jiménez et al., 2020), en ambientes urbanizados de Guerrero (Rodríguez-Rodríguez et al., 2015), Tamaulipas (Salazar-Olivo \& Solís-Rojas, 2015) y Michoacán (Maldonado-Carrizales \& Ponce-Saavedra, 2017; Maldonado-Carrizales et al., 2018), en las orillas de un río en Hidalgo (Cipriano-Anastasio et al., 2019) y en bosques tropicales de San Luis Potosí (Rivera-Quiroz et al., 2016).

Para el Estado de México existen trabajos taxonómicos que incluyen la descripción y registro de varias especies (Platnick \& Shadab, 1975; 1977; 1980; 1982; Desales-Lara, 2012). Sin embargo, son casi nulos los trabajos en aspectos ecológicos, solo se cuenta con las investigaciones de Desales-Lara et al. (2013) en ambientes urbanos, y el de Campuzano y Padilla-Ramírez (2020) en dos remanentes de bosque de pino-encino. Adicionalmente, se tiene información proveniente de los listados sobre Araneae de Hoffmann (1976) y Desales-Lara (2014). En este último, se citan 122 especies pertenecen al clado RTA, y de éstas 22 se registraron en el municipio de Tonatico. Recientemente Guerrero-Fuentes y Desales-Lara (2020) dan a conocer nuevos registros de arañas para el estado y país con ejemplares recolectados en dicho municipio.

Aunque los trabajos anteriores registran especies del clado RTA en el país, solo la investigación de Campuzano y Padilla-Ramírez (2020) trata la ecología de estas arañas en particular. Por tal motivo, el presente trabajo se enfocó en evaluar la abundancia, riqueza, diversidad, composición y estructura de las comunidades de estas arañas entre lluvias y secas en tres tipos de vegetación presentes en Tonatico, Estado de México.

\section{MATERIALES Y MÉTODOS}

Área de estudio. En la parte sur de Tonatico, Estado de México (Fig. 1), se establecieron tres sitios de recolecta con base en los tipos de vegetación indicados por el INEGI (2009) y por Contreras et al. (2010). La Vega (18 46' 19.67" N, 99 39' 21.81" O, 1,614 m s.n.m.), con vegetación correspondiente a un pastizal inducido (PI), este lugar presenta un alto grado de perturbación, producto de las actividades ganaderas y agrícolas que allí se realizan (obs. pers.); San Bartolo (18 
48' 22.43" N, 99 37' 36.44" O, 1,678 m s.n.m.), cuya vegetación es un bosque de pino (BP); y La Audiencia (18 $49^{\prime} 1.27^{\prime \prime} \mathrm{N}, 99^{\circ} 37^{\prime} 13.58^{\prime \prime} \mathrm{O}, 1,634 \mathrm{~m}$ s.n.m.) correspondiente a una selva baja caducifolia (SBC). En la zona predomina un clima tipo AC (w) semicálido con lluvias en verano que van de finales de mayo a principios de octubre, alcanzando al año entre los $800 \mathrm{~mm}$ y los 1,100 $\mathrm{mm}$ de precipitación pluvial (Marmolejo, 2010). El promedio de temperatura varía entre 18 y $24^{\circ} \mathrm{C}$. La temperatura más elevada ocurre durante los meses de abril y mayo, mientras que las temperaturas más bajas se registran en los meses de noviembre, diciembre y enero (Vázquez, 1999; Marmolejo, 2010).

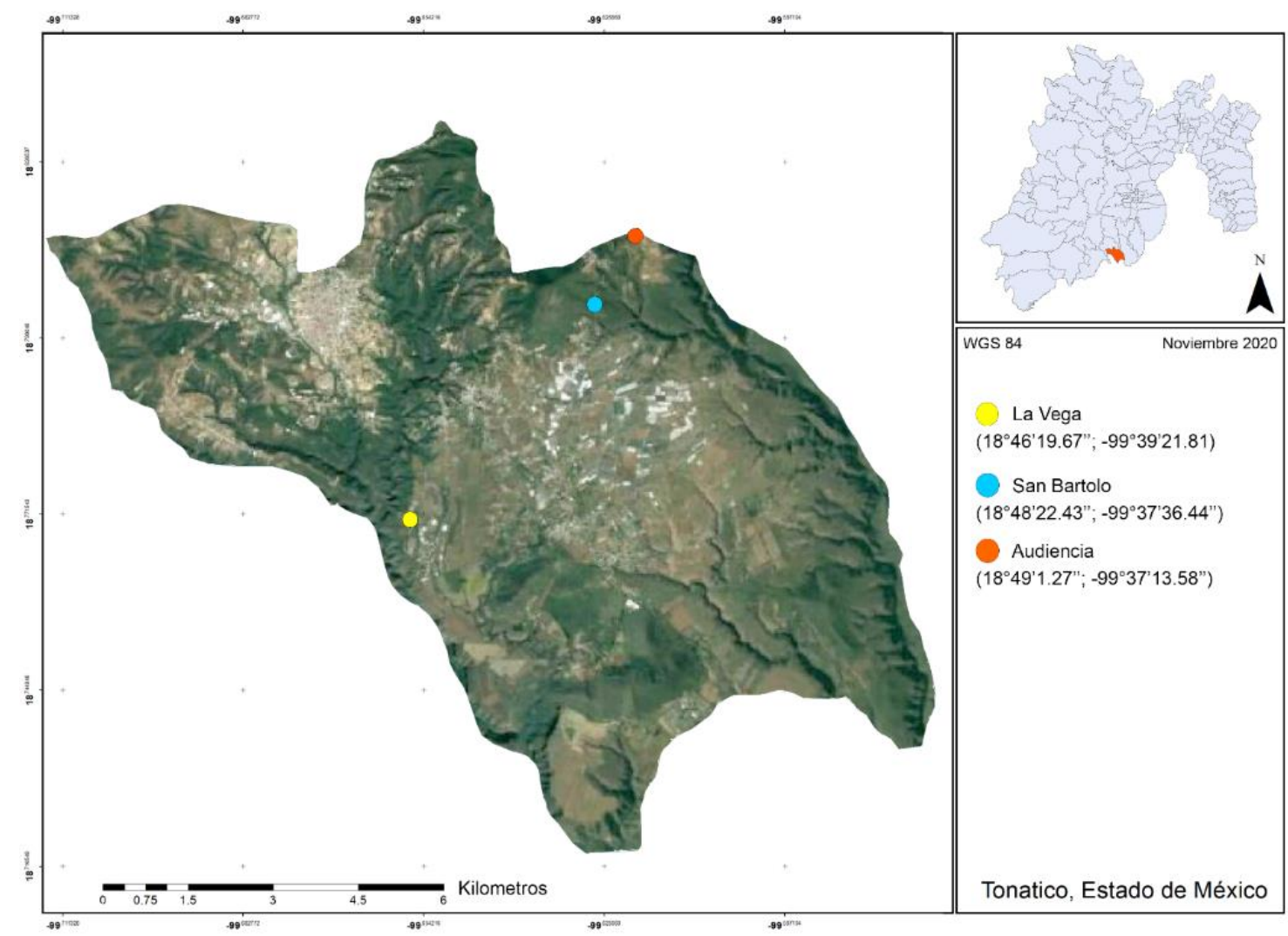

Figura 1. Ubicación de los sitios de muestreo de arañas dentro del municipio de Tonatico, Estado de México.

Trabajo de Campo. En cada sitio de recolecta se delimitó un área de $300 \mathrm{~m}^{2}$ en donde se dispusieron diez trampas de caída, sobre un transecto de 50 metros de largo, las trampas se encontraban distribuidas cinco a la izquierda y cinco a la derecha, separadas una de otra por diez metros a lo largo y seis metros a lo ancho Fig. 2). Cada trampa consistió en un contenedor de plástico de $10 \mathrm{~cm}$ de diámetro de apertura y capacidad de un litro enterrado a nivel del suelo y una tapa de plástico de $15 \mathrm{~cm}$ de diámetro que evitó la acumulación excesiva de agua y hojarasca. Entre la boca del recipiente y la tapa se dejaron aproximadamente cinco centímetros de separación. Los recipientes fueron llenados a un tercio de su capacidad con una mezcla de $250 \mathrm{ml}$ de alcohol etílico al 70\% como conservador y $85 \mathrm{ml}$ de monoetilenglicol para evitar la evaporación. 
Las trampas permanecieron activas de noviembre del 2011 a octubre del 2012, durante ese periodo las trampas fueron revisadas una vez por mes. El material recolectado fue etiquetado y transportado dentro de bolsas herméticas con alcohol etílico al 70\%, al Centro de Investigación en Recursos Bióticos (CIRB) de la Universidad Autónoma del Estado de México (UAEMex).

Trabajo de gabinete. Las arañas del clado RTA se separaron del resto de los artrópodos con ayuda de un microscopio estereoscópico Olympus modelo SD-ILK200-2 y se preservaron en alcohol al $70 \%$. Los especímenes adultos y juveniles se determinaron a nivel de familia con la clave de arañas de Norte América (Ubick et al., 2017). Los adultos se determinaron a nivel de género y especie con claves y publicaciones específicas. Además, para realizar las determinaciones a nivel de especie se disectó la genitalia de algunos ejemplares adultos y se siguió el protocolo de Guerrero-Fuentes y Francke (2019) para el aclarado de estructuras, usando $\mathrm{KOH}$ al $10 \%$ y aceite de clavo. Todos los organismos recolectados se depositaron en la Colección de Arácnidos del Estado de México (CAEM) de la UAEMex.

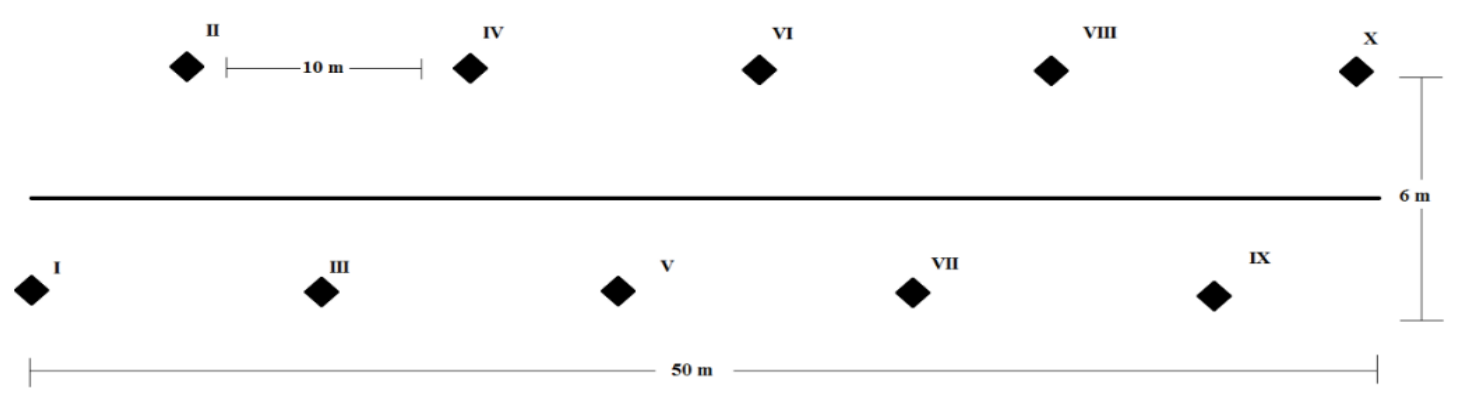

Figura 2. Distribución espacial de diez trampas de caída en cada uno de los sitos de muestreo.

\section{Análisis estadísticos}

Abundancias. Los análisis de abundancia fueron los únicos en los que se consideraron los ejemplares juveniles; en el resto de las pruebas, solo se tomaron en cuenta a los ejemplares adultos. Para cada sitio, los datos de abundancia se obtuvieron al sumar el total de adultos y juveniles encontrados por trampa, sin considerar la identidad taxonómica de los ejemplares; posteriormente esas cifras fueron sumadas nuevamente para cada mes. Los datos de abundancia fueron comparados mediante pruebas no paramétricas, utilizadas en problemas cuyos datos provienen de una escala ordinal, en dos ejercicios (Márquez, 2004). En el primero se realizó una prueba de Kruskal-Wallis con los datos separados por sitio para todo el año, considerando a cada mes como una observación. En el segundo ejercicio se hizo una $\mathrm{U}$ de Mann-Whitney dividiendo las observaciones por temporada de lluvias (mayo-octubre) y secas (noviembre-abril).

Riqueza y Diversidad alfa. Para estimar la riqueza de cada tipo de vegetación utilizamos un análisis de rarefacción con base en el número de individuos en el programa Estimates 9.1 (Colwell, 2013). La riqueza rarefaccionada se comparó mediante la sobreposición de los valores de los intervalos de confianza al 95\% (López-Mejía et al., 2017). Se obtuvo el índice de entropía de Shannon-Wiener $\left(\mathrm{H}^{\prime}\right)$ por temporada climática y para todo el año en cada zona muestreada. 
Posteriormente, se utilizaron pruebas de $t$-Hutchinson para detectar diferencias significativas en los valores de $\mathrm{H}^{\prime}$ entre temporadas y entre ecosistemas (Pinkus et al., 2006).

Para conocer los cambios en la diversidad entre temporadas y tipos de vegetación, se calculó el número efectivo de especies $\left({ }^{9} \mathrm{D}\right)$, que son las unidades de medición de la diversidad verdadera. Esta aproximación permite comparar en unidades de especies la diversidad descrita por otros índices de diversidad (Moreno et al., 2011). En este trabajo, 9D se calculó usando los valores de $\mathrm{H}^{\prime}$ y un exponente $q=1$, donde todas las especies son incluidas con un peso exactamente proporcional a su abundancia en la comunidad y por medio de la ecuación expresada como ${ }^{1} D=\exp \left(H^{\prime}\right)$ (Jost, 2006).

La comparación por pares de la diversidad verdadera entre comunidades, expresada en magnitud del número de especies de diferencia entre un sitio y otro, se hizo tomando en cuenta que la comunidad $A$ (la más diversa de acuerdo con $\mathrm{H}^{\prime}$ ) tiene una diversidad $D_{A}$ mayor que la diversidad $D_{B}$ de la comunidad $B$, entonces la comunidad $A$ tuvo $D_{A} D_{B}$ veces más diversidad que la comunidad $B$, expresado de otra manera, la comunidad $B$ alcanzó un porcentaje $\left(D_{B} \times 100\right) / D_{A}$ de la diversidad de la comunidad $A$ (Moreno et al., 2011).

Integridad de los inventarios. Para evaluar la fiabilidad e integridad de los inventarios para cada sitio, por temporada climática y durante todo el año, se dividió la riqueza total observada contra la riqueza estimada por Chao 1 con el programa EstimateS 9.1. El resultado expresado en porcentaje se tomó como medida para determinar qué tan completo fue el inventario en cada caso (Maya-Morales et al., 2012).

Composición de especies. Para probar si había diferencias en la composición de arañas entre cada tipo de vegetación, se empleó un análisis unidireccional (ANOSIM) con el índice de Bray-Curtis (abundancia de las especies). Además, se empleó un análisis de agrupamiento con el mismo índice y el método de grupo de pares no ponderados con media aritmética (UPGMA) para establecer la similitud entre los sitios de colecta. Ambos análisis se realizaron con el programa PAST 3.24 (Hammer et al., 2001).

Estructura de la comunidad. Se calculó la medida de complementariedad de Colwell y Coddington (1994), esta medida expresa el grado de disimilitud en la composición de especies entre pares de biotas. El índice se obtiene con la fórmula $C_{A B}=U A B / S A B$, la cual se calcula a través de la riqueza total para los sitios combinados, $S A B=a+b-c$ (donde $a$ es el número de especies del sitio $A$, mientras $b$ es el número de especies del sitio $B$ y $c$ el número de especies en común entre los dos sitios) y de la riqueza en conjunto de especies exclusivas de cada sitio $U_{A B}=$ $a+b-2 c$. El valor obtenido varía desde cero cuando ambos sitios son idénticos en cuanto a la composición de especies, hasta uno cuando las especies de cada sitio son completamente distintas (Moreno, 2001). Además, se obtuvo la dominancia para cada uno de los sitios con el índice de Simpson $(\lambda)$. Posteriormente, se realizaron curvas de rango-abundancia para fijar visualmente la dominancia $(\lambda)$ de las especies entre los diferentes tipos de vegetación (James \& Rathbun, 1981). Por último, se hicieron categorizaciones ecológicas con la prueba de asociación no métrica de Olmstead-Tukey, esta prueba permite clasificar a las especies de forma gráfica dentro de cuatro 
categorías establecidas en función del logaritmo de la abundancia (log $X+1)$ y la frecuencia de aparición, la delimitación de los cuadrantes se establece tomando los valores promedio de los datos graficados en cada eje (Sokal \& Rohlf, 1981). En este estudio, en el eje de las ordenadas, se empleó la modificación propuesta por Desales-Lara et al., (2013), en la cual el número de meses que dura el estudio se divide entre dos. La categoría de las especies se estableció bajo la propuesta de Maya-Morales et al. (2012), donde las especies se pudieron ubicar en una de las siguientes categorías: dominantes (abundantes y frecuentes), temporales (abundantes y poco frecuentes), constantes (poco abundantes y frecuentes) y raras (poco abundantes y poco frecuentes).

\section{RESULTADOS \\ Abundancia y riqueza}

Se recolectó un total de 805 arañas (230 $152+$ y 423 juveniles), las cuales se agruparon en 16 familias, 29 géneros y 49 especies (25 determinadas a nivel específico, 21 a género y tres morfoespecies) (Cuadro 1). Las familias más abundantes fueron Lycosidae (41\%), Gnaphosidae (13\%), Salticidae (11\%) y Corinnidae (8\%). Las especies, Pardosa medialis Banks, 1898, P. pauxilla Montgomery, 1904 y P. marialuisae Dondale \& Redner, 1984 representaron el 38\% del total de la muestra (Cuadro 1). En el BP se encontraron 444 individuos (55\% de la muestra global) de los cuales, 223 fueron adultos. En el PI se recolectaron 189 organismos ( $24 \%$ de la muestra total), 72 fueron adultos. Mientras que en la SBC se hallaron 172 ejemplares ( $21 \%$ del total) de estos, 87 eran adultos. Al comparar las abundancias totales (Cuadro 2) para los tres sitios, la prueba de Kruskal-Wallis no indicó diferencias significativas $(H=2.347, d f=2, p=0.3084$. Fig. 3$)$. Respecto a la influencia de las temporadas de secas y lluvias sobre la abundancia total de arañas (Cuadro $2)$, las pruebas de $U$ Mann-Whitney no mostraron diferencias significativas en la $\operatorname{SBC}(z=\infty, p=$ $1)$, el BP $(z=0.523, p=0.127)$ y el PI $(z=0.482, p=0.629)$, ni para el área total de estudio $(z=$ 1.521, $p=0.128$. Fig. 4).

Cuadro 1. Abundancia de las especies y familias recolectadas. El número entre paréntesis al lado del nombre de cada familia indica la abundancia de ejemplares incluyendo juveniles. PI: Pastizal Inducido, BP: Bosque de Pino, SBC: Selva Baja Caducifolia y Ab: abundancia de adultos. El número entre paréntesis indíca el número de meses en los que se registró la especie.

\begin{tabular}{llllll}
\hline Familia & Especie & $\begin{array}{l}\mathbf{P I} \\
\mathbf{A b} \text { (n }{ }^{\circ} \text { meses) }\end{array}$ & $\begin{array}{l}\mathbf{B P} \\
\mathbf{A b} \text { (n }{ }^{\circ} \text { meses) }\end{array}$ & $\begin{array}{l}\text { SBC } \\
\mathbf{A b} \text { (n }{ }^{\circ} \text { meses) }\end{array}$ & $\begin{array}{l}\text { Total adultos Ab } \\
\left(\mathbf{n}^{\circ} \text { meses) }\right.\end{array}$ \\
\hline Agelenidae (1) & Melpomene elegans & 0 & 0 & $1(1)$ & $1(1)$ \\
\hline Anyphaenidae (51) & Anyphaena gibbosa & 0 & $2(1)$ & $1(1)$ & $3(1)$ \\
& Anyphaena sp. & $1(1)$ & 0 & 0 & $1(1)$ \\
\hline Clubionidae (6) & Clubiona sp. & $3(3)$ & $2(2)$ & 0 & $5(4)$ \\
\hline Corinnidae (68) & Castianeira azteca & 0 & $6(4)$ & $10(3)$ & $16(5)$ \\
& Castianeira sp. 1 & 0 & $2(2)$ & $5(2)$ & $7(3)$ \\
& Castianeira sp. 2 & 0 & $1(1)$ & 0 & $1(1)$ \\
& Castianeira sp. 3 & 0 & $4(2)$ & $1(1)$ & $5(2)$ \\
& Castianeira stylifera & $1(1)$ & $1(1)$ & $5(2)$ & $7(2)$ \\
& Creugas gulosus & 0 & $3(2)$ & $3(2)$ & $6(3)$ \\
& Creugas sp. & 0 & 0 & $3(2)$ & $3(2)$ \\
\hline
\end{tabular}




\begin{tabular}{|c|c|c|c|c|c|}
\hline Familia & Especie & $\begin{array}{l}\mathrm{PI} \\
\mathrm{Ab} \text { ( } \mathrm{n}^{\circ} \text { meses) }\end{array}$ & $\begin{array}{l}\text { BP } \\
\text { Ab ( } n^{\circ} \text { meses) }\end{array}$ & $\begin{array}{l}\text { SBC } \\
A b \text { ( } n^{\circ} \text { meses) }\end{array}$ & $\begin{array}{l}\text { Total adultos Ab } \\
\left(n^{\circ} \text { meses) }\right.\end{array}$ \\
\hline Ctenidae (22) & Leptoctenus sp. & $1(1)$ & $1(1)$ & $7(3)$ & $9(4)$ \\
\hline \multirow[t]{5}{*}{ Gnaphosidae (102) } & Cesonia cuernavaca & 0 & 0 & $13(4)$ & $13(4)$ \\
\hline & Cesonia lugubris & $3(3)$ & $10(4)$ & $6(4)$ & $19(7)$ \\
\hline & Drassyllus sp. & $2(2)$ & $6(1)$ & $3(1)$ & $11(2)$ \\
\hline & Herpyllus brachet & 0 & $1(1)$ & $1(1)$ & $2(2)$ \\
\hline & Herpyllus sp. & 0 & $2(2)$ & $1(1)$ & $3(3)$ \\
\hline \multirow[t]{9}{*}{ Lycosidae (330) } & Alopecosa sp. & $1(1)$ & 0 & 0 & $1(1)$ \\
\hline & Hogna sp. & $4(4)$ & $4(2)$ & 0 & $8(5)$ \\
\hline & Lycosidae sp. 1 & 0 & 0 & $3(2)$ & $3(2)$ \\
\hline & Lycosidae sp. 2 & $2(2)$ & 0 & 0 & $2(2)$ \\
\hline & Pardosa marialuisae & $3(1)$ & $27(2)$ & 0 & $30(2)$ \\
\hline & Pardosa medialis & $12(5)$ & $55(7)$ & $1(1)$ & $68(8)$ \\
\hline & Pardosa pauxilla & $8(2)$ & $38(7)$ & $1(1)$ & $47(7)$ \\
\hline & Pardosa sp. 1 & $1(1)$ & $2(1)$ & 0 & $3(1)$ \\
\hline & Pardosa sp. 2 & 0 & $1(1)$ & 0 & $1(1)$ \\
\hline \multirow[t]{2}{*}{ Oxyopidae (50) } & Oxyopes sp. 1 & 0 & $16(4)$ & 0 & $16(4)$ \\
\hline & Oxyopes sp. 2 & 0 & $8(2)$ & $1(1)$ & $9(2)$ \\
\hline Philodromidae (19) & No determinados & 0 & 0 & 0 & 0 \\
\hline Pisauridae (2) & Pisauridae sp. & 0 & $2(1)$ & 0 & $2(1)$ \\
\hline \multirow[t]{13}{*}{ Salticidae (90) } & Colonus hesperus & 0 & $1(1)$ & $1(1)$ & $2(1)$ \\
\hline & Habronattus fallax & $8(4)$ & $6(4)$ & $1(1)$ & $15(9)$ \\
\hline & Habronattus nahuatlanus & $8(2)$ & $1(1)$ & $1(1)$ & $10(4)$ \\
\hline & Habronattus mexicanus & $1(1)$ & $4(4)$ & 0 & $5(4)$ \\
\hline & Marpissa pikei & 0 & $1(1)$ & 0 & $1(1)$ \\
\hline & Phanias sp. & 0 & 0 & $1(1)$ & $1(1)$ \\
\hline & Mexigonus minutus & 0 & $1(1)$ & 0 & $1(1)$ \\
\hline & Corythalia sp. & 0 & $1(1)$ & $7(2)$ & $8(3)$ \\
\hline & Sassacus alboguttatus & 0 & $1(1)$ & $1(1)$ & $2(2)$ \\
\hline & Peckhamia americana & $5(3)$ & 0 & 0 & $5(3)$ \\
\hline & Peckhamia sp. & 0 & $1(1)$ & 0 & $1(1)$ \\
\hline & Phidippus sp. & 0 & $1(1)$ & 0 & $1(1)$ \\
\hline & $\begin{array}{l}\text { Metacyrba taeniola } \\
\text { taeniola }\end{array}$ & 0 & $1(1)$ & 0 & $1(1)$ \\
\hline Selenopidae (3) & Selenops sp. & 0 & 0 & $2(2)$ & $2(2)$ \\
\hline Sparassidae (5) & Curicaberis sp. & 0 & 0 & $1(1)$ & $1(1)$ \\
\hline \multirow[t]{2}{*}{ Thomisidae (41) } & Xysticus paiutus & $3(2)$ & $4(2)$ & 0 & $7(3)$ \\
\hline & Xysticus pellax & 0 & $2(2)$ & 0 & $2(2)$ \\
\hline \multirow[t]{2}{*}{ Trachelidae (7) } & Meriola decepta & $3(3)$ & $1(1)$ & 0 & $4(3)$ \\
\hline & Trachelas depressus & $2(2)$ & $1(1)$ & 0 & $3(3)$ \\
\hline \multirow[t]{3}{*}{ Zoropsidae (8) } & Zorocrates fuscus & 0 & $2(2)$ & $6(6)$ & $8(3)$ \\
\hline & Abundancia adultos & 72 & 223 & 87 & 382 \\
\hline & Riqueza & 20 & 38 & 27 & 49 \\
\hline
\end{tabular}


El BP presentó el 78\% de la riqueza, la SBC el 55\% y el PI el $41 \%$ (Cuadro 1). El análisis de rarefacción de especies (Fig. 5) indicó que no existen diferencias significativas entre la riqueza rarefaccionada de cada sitio al sobreponerse los intervalos de confianza. De acuerdo con la temporada climática para toda el área de estudio, la época de lluvias presentó un $86 \%$ de la riqueza y la temporada de secas el $53 \%$ del total de las especies registradas. En los sitios del BP y la SBC se observó casi el doble de riqueza de especies comparado con la temporada de secas; mientras que, en el PI sólo hubo una especie de diferencia entre temporadas (Cuadro 3).

\section{Integridad de los inventarios}

Para el ciclo anual, el inventario del PI fue el más completo al presentar el $84 \%$ de la diversidad estimada por Chao 1, seguido del BP con 74\% y la SBC 38\%. Al considerar la temporada de lluvias, los valores de integridad fueron superiores al $70 \%$ en todos los sitios; además, el PI fue el sitio mejor representado (84\%). Para la época de sequía, en el PI y la SBC la integridad de los inventarios fue del 60\%; el inventario del BP presentó solo $21 \%$ de las especies estimadas (Cuadro 4).

Cuadro 2. Abundancia total de arañas (adultos y juveniles) del clado RTA recolectadas en cada sitio y por mes de muestreo. PI: Pastizal Inducido, BP: Bosque de Pino y SBC: Selva Baja Caducifolia.

\begin{tabular}{llllllllllllll}
\hline & \multicolumn{1}{c}{ Lluvias } & \multicolumn{1}{c}{ Secas } \\
\hline & May & Jun & Jul & Ago & Sep & Oct & Nov & Dic & Ene & Feb & Mar & Abr & Total \\
\hline PI & 29 & 2 & 35 & 19 & 16 & 0 & 4 & 0 & 13 & 5 & 5 & 61 & 189 \\
BP & 9 & 42 & 83 & 10 & 133 & 40 & 76 & 19 & 8 & 11 & 5 & 8 & 444 \\
SBC & 8 & 10 & 26 & 27 & 16 & 9 & 9 & 13 & 14 & 17 & 12 & 11 & 172 \\
General & 46 & 54 & 144 & 56 & 165 & 49 & 89 & 32 & 35 & 33 & 22 & 80 & 805 \\
\hline
\end{tabular}

Cuadro 3. Riqueza de especies (S), índice de Shannon ( $\left.\mathrm{H}^{\prime}\right)$ y número efectivo de especies (1D) para cada tipo de vegetación por temporada climática y para todo el año. PI: Pastizal Inducido, BP: Bosque de Pino y SBC: Selva Baja Caducifolia.

\begin{tabular}{llllllllll}
\hline & PI & & \multicolumn{3}{c}{ BP } & \multicolumn{3}{c}{ SBC } \\
\hline & Lluvias & Secas & General & Lluvias & Secas & General & Lluvias & Secas & General \\
\hline $\mathrm{S}$ & 15 & 14 & 20 & 32 & 13 & 38 & 22 & 11 & 27 \\
$\mathrm{H}^{\prime}$ & 2.19 & 2.15 & 2.69 & 2.47 & 1.85 & 2.73 & 2.59 & 1.93 & 2.89 \\
${ }^{1} \mathrm{D}$ & 8.93 & 8.58 & 14.73 & 11.82 & 6.36 & 15.33 & 13.33 & 6.89 & 17.99 \\
\hline
\end{tabular}

Cuadro 4. Valores de la abundancia de arañas adultas $(\mathrm{N})$, especies observadas (S), especies estimadas por Chao 1, integridad del inventario en porcentaje ( $\mathrm{S} / \mathrm{Chao1}$ ) para cada tipo de vegetación y por temporada climática. PI: Pastizal Inducido. BP: Bosque de Pino. SBC: Selva Baja Caducifolia.

\begin{tabular}{llllllllll}
\hline & \multicolumn{3}{l}{ Temporada seca } & \multicolumn{2}{l}{ Temporada de lluvias } & Año \\
\hline & $\mathrm{PI}$ & $\mathrm{BP}$ & $\mathrm{SBC}$ & $\mathrm{PI}$ & $\mathrm{BP}$ & $\mathrm{SBC}$ & $\mathrm{PI}$ & $\mathrm{BP}$ & $\mathrm{SBC}$ \\
\hline $\mathrm{N}$ & 29 & 32 & 24 & 43 & 191 & 63 & 72 & 223 & 87 \\
$\mathrm{~S}$ & 14 & 14 & 11 & 15 & 32 & 22 & 20 & 38 & 27 \\
Chao 1 & 21 & 67 & 18 & 18 & 41 & 30 & 24 & 51 & 72 \\
S/Chao 1 & $67 \%$ & $21 \%$ & $60 \%$ & $84 \%$ & $79 \%$ & $73 \%$ & $84 \%$ & $74 \%$ & $38 \%$ \\
\hline
\end{tabular}




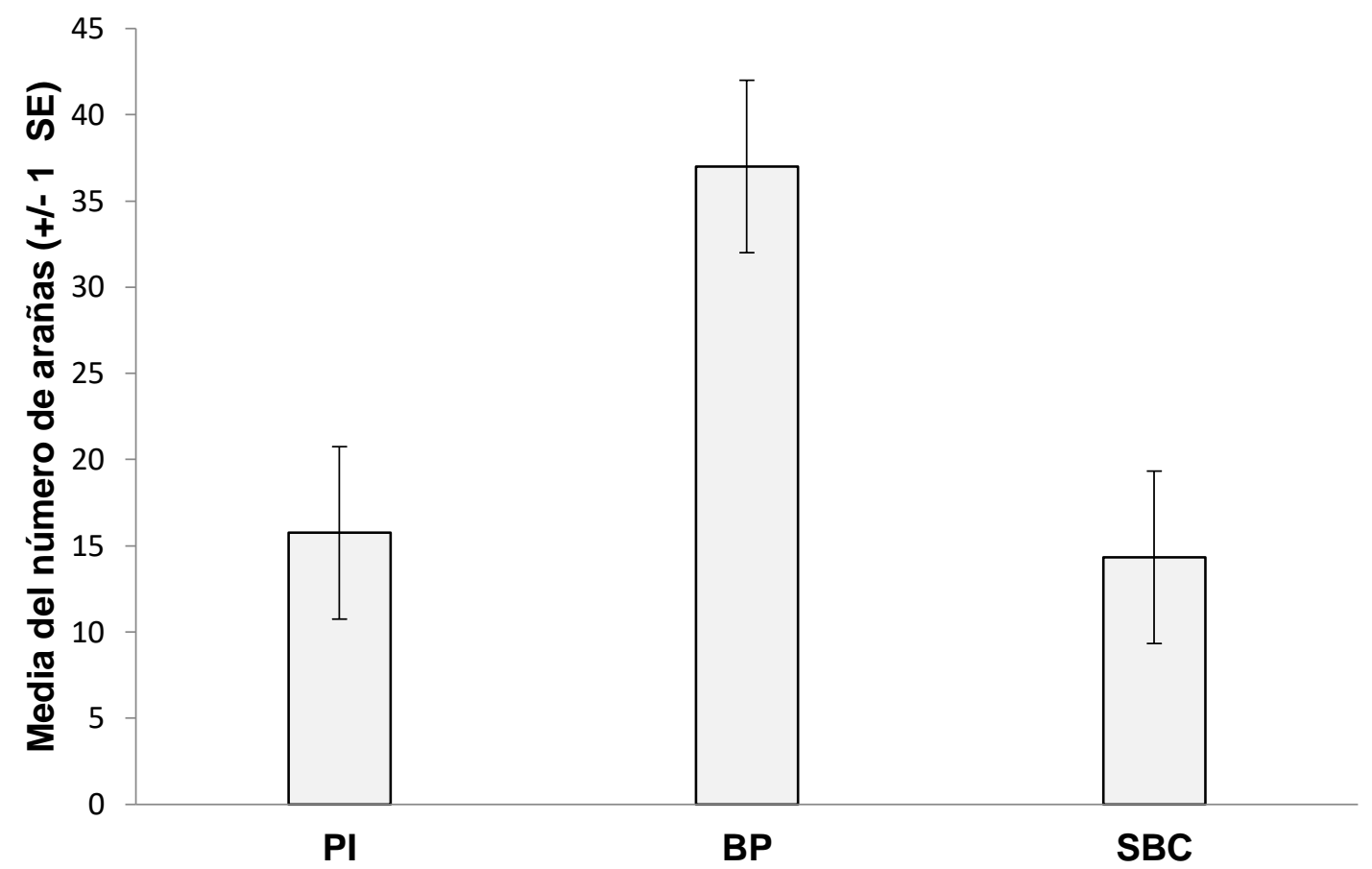

Figuras 3. Abundancias promedio del total de arañas recolectadas por sitio durante un año. Las barras de error corresponden al error estándar (SE) de las abundancias promedio. PI: Pastizal Inducido, BP: Bosque de Pino y SBC: Selva Baja Caducifolia.

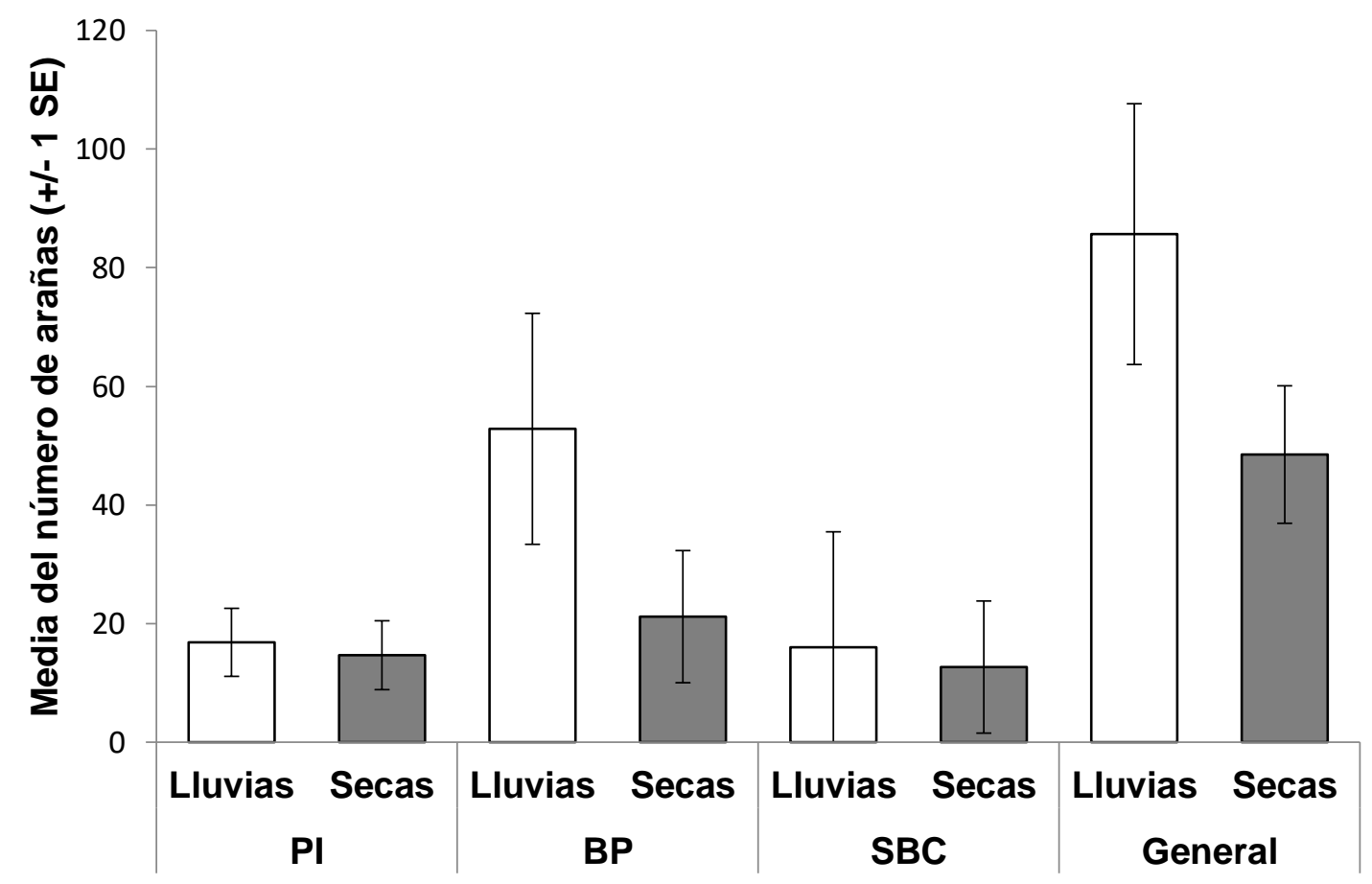

Figura 4. Abundancias promedio del total de arañas recolectadas por temporada climática en cada sitio. Las barras de error corresponden al error estándar (SE) de las abundancias promedio. Pl: Pastizal Inducido, BP: Bosque de Pino y SBC: Selva Baja Caducifolia. 


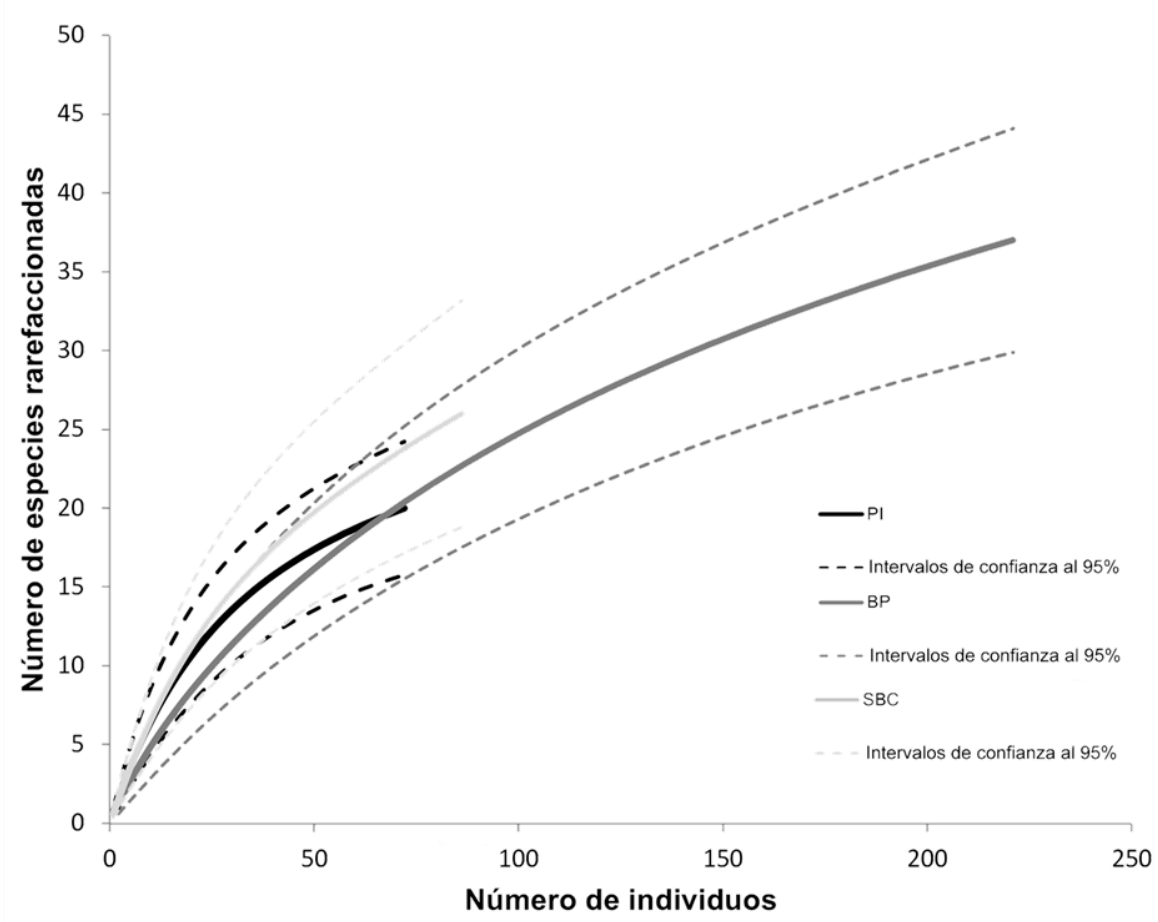

Figura 5. Riqueza de especies rarefaccionada para cada sitio de recolecta. Las líneas punteadas corresponden a los intervalos de confianza al 95\%. PI: Pastizal Inducido, BP: Bosque de Pino y SBC: Selva Baja Caducifolia.

\section{Diversidad alfa}

Al comparar entre los sitios la diversidad alfa alcanzada en el año (Cuadro 3), las pruebas de $t$ Hutchinson basadas en el índice de Shannon-Wiener $\left(\mathrm{H}^{\prime}\right)$ no encontraron diferencias significativas (PI vs BP $t=-0.73, d f=194.34, p=0.930 ; \mathrm{BP}$ vs SBC $t=-0.6, d f=204.24, p=0.560 ; \mathrm{SBC}$ vs $\mathrm{PI} t=$ $-1.25, d f=154.99, p=0.220$ ). De acuerdo con el número efectivo de especies, la SBC obtuvo una diferencia de 1.22 y 1.17 más especies que el PI y el BP, respectivamente, en otras palabras, el PI y el BP presentaron el $82 \%$ y $85 \%$ de la diversidad de la SBC (Cuadro 3). Al contrastar la diversidad de arañas $\left(H^{\prime}\right)$ entre temporadas climáticas (lluvias vs secas) para cada sitio (Cuadro 3 ), se encontraron diferencias significativas en el BP $(t=2.69, d f=41.82, p=0.010)$ y la SBC $(t=3.2, d f$ $=43.85$. $p=0.002)$, en tanto que en el PI $(t=0.18, d f=64.8, p=0.857)$ no se hallaron diferencias significativas. De acuerdo con el número efectivo de especies (Cuadro 3), todos los sitios fueron más diversos durante la temporada húmeda en comparación con el periodo de secas. El PI tuvo 1.04 más especies en lluvias, es decir, durante la temporada seca se alcanzó el $96 \%$ de la diversidad presente en lluvias. En el BP hubo una diferencia de 1.86 especies entre temporadas y en el periodo seco se registró el $58.8 \%$ de la diversidad de la temporada húmeda. Mientras, en la SBC se encontró 1.93 especies de diferencia y en secas se alcanzó el 51.7\% de la diversidad de la época de lluvias.

\section{Composición de especies y estructura de la comunidad}


De las 49 especies encontradas en el estudio, $43 \%$ fueron exclusivas de un sitio (BP = diez especies, $\mathrm{SBC}=$ siete especies y $\mathrm{PI}=$ cuatro especies), $41 \%$ se recolectaron en dos de las localidades y $16 \%$ se hallaron en los tres sitios de muestreo (Cuadro 1). La composición de arañas entre los tipos de vegetación fue significativamente diferente de acuerdo con la prueba ANOSIM $(R=0.1288, p=$ 0.001). Los valores de significancia del análisis de similitudes (ANOSIM) entre los sitios indicaron que entre el BP y el PI no hay diferencia significativa $(R=0.0576, p=0.1064)$, por lo tanto, hay mayor similitud entre estos sitios (Fig. 6). En la comparación por pares de los sitios, la medida de complementariedad $\left(C_{A B}\right)$ presentó valores mayores al 0.55 de diferencia en la composición de arañas del clado RTA. El PI y la SBC fueron los sitios con el recambio de especies más elevado $\left(C_{A B}\right.$ $=0.79$; ocho especies compartidas), seguido del par PI vs $B P\left(C_{A B}=0.62\right.$ y 16 especies compartidas), por último, el conjunto $\mathrm{SBC} v s \mathrm{BP}\left(\mathrm{C}_{A B}=0.56\right.$ y 20 especies compartidas).

Las curvas de rango abundancia (Fig. 7) muestran que la dominancia fue alta en la SBC y el $\mathrm{PI}$, el BP fue más equitativo, lo anterior concuerda con lo obtenido por el índice de Simpson, donde la SBC presento el valor de dominancia más alto $(\lambda=0.92)$, seguido del PI $(\lambda=0.91)$, y por último en BP $(\lambda=0.89)$. En general, las especies más frecuentes durante el año y para todo el estudio fueron: Habronattus fallax Peckham \& Peckham, 1909 registrada durante nueve meses, $P$. medialis en ocho meses, P. pauxilla y Cesonia lugubris O. Pickard-Cambridge, 1896 se presentaron durante siete meses (Cuadro 1).

Respecto a la categoría ecológica, en ninguno de los sitios se detectaron especies constantes (Cuadro 5). Durante la temporada de lluvias, Castianeira stylifera Kraus, 1955 se categorizó como una especie temporal en la SBC, rara en el PI y el BP; C. lugubris fue dominante en la SBC, temporal en el $\mathrm{BP}$ y rara en el $\mathrm{Pl} ; \mathrm{P}$. medialis fue dominante en el $\mathrm{BP}$ y el $\mathrm{Pl}$, mientras que en la SBC fue rara; por último, P. pauxilla fue dominante en el BP, temporal en el PI y rara en la SBC (Cuadro 5).

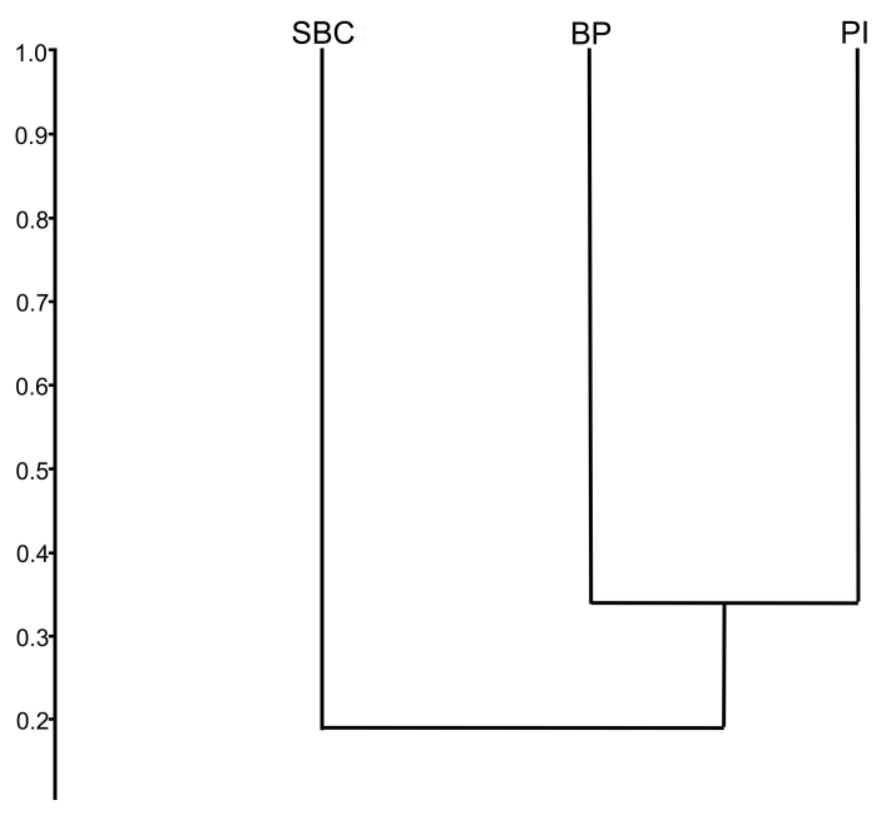


Figura 6. Análisis de agrupamiento con base en el índice de Bray-Curtis (abundancias de las especies) de los sitios muestreados. PI: Pastizal Inducido, BP: Bosque de Pino y SBC: Selva Baja Caducifolia.

Cuadro 5. Categoría ecológica alcanzada por cada especie, R: raras, T: temporales, D: dominantes y N: no encontrada. Las categorías se presentan para todo el año (Td), para las lluvias (LL) y para las secas(S). Pl: Pastizal Inducido, BP: Bosque de Pino y SBC: Selva Baja Caducifolia.

\begin{tabular}{|c|c|c|c|}
\hline Especie & $\begin{array}{l}\text { PI } \\
\text { Td (LL, S) }\end{array}$ & $\begin{array}{l}\text { BP } \\
\text { Td }(L L, S) \\
\end{array}$ & $\begin{array}{l}\text { SBC } \\
\text { Td }(L L, S)\end{array}$ \\
\hline Melpomene elegans & & & $R(R, N)$ \\
\hline Anyphaena gibbosa & & $\mathrm{R}(\mathrm{R}, \mathrm{N})$ & $R(R, N)$ \\
\hline Anyphaena sp. & $R(R, N)$ & & \\
\hline Clubiona sp. & $T(T, R)$ & & \\
\hline Castianeira azteca & & $T(D, N)$ & $T(D, N)$ \\
\hline Castianeira sp. 1 & & $R(R, N)$ & $T(T, R)$ \\
\hline Castianeira sp. 2 & & $R(R, N)$ & \\
\hline Castianeira sp. 3 & & $\mathrm{~T}(\mathrm{~T}, \mathrm{~N})$ & $R(R, N)$ \\
\hline Castianeira stylifera & $R(R, N)$ & $R(R, N)$ & $T(T, R)$ \\
\hline Creugas gulosus & & $T(R, R)$ & $\mathrm{T}(\mathrm{N}, \mathrm{T})$ \\
\hline Creugas sp. & & & $\mathrm{T}(\mathrm{N}, \mathrm{T})$ \\
\hline Leptoctenus sp. & $R(N, R)$ & $R(N, R)$ & $\mathrm{T}(\mathrm{T}, \mathrm{T})$ \\
\hline Cesonia cuernavaca & & & $\mathrm{T}(\mathrm{T}, \mathrm{T})$ \\
\hline Cesonia lugubris & $T(R, R)$ & $T(T, R)$ & $T(D, R)$ \\
\hline Drassyllus sp. & $R(N, R)$ & $\mathrm{T}(\mathrm{T}, \mathrm{N})$ & $T(T, N)$ \\
\hline Herpyllus brachet & & $R(N, R)$ & $R(R, N)$ \\
\hline Herpyllus sp. & & $R(R, R)$ & $R(R, N)$ \\
\hline Alopecosa sp. & $R(N, R)$ & & \\
\hline Hogna sp. & $T(R, R)$ & $\mathrm{T}(\mathrm{N}, \mathrm{T})$ & \\
\hline Lycosidae sp. 1 & & & $\mathrm{~T}(\mathrm{~N}, \mathrm{~T})$ \\
\hline Lycosidae sp. 2 & $R(R, R)$ & & \\
\hline Pardosa marialuisae & $\mathrm{T}(\mathrm{T}, \mathrm{N})$ & $\mathrm{T}(\mathrm{T}, \mathrm{N})$ & \\
\hline Pardosa medialis & $T(D, R)$ & $D(D, R)$ & $R(R, N)$ \\
\hline Pardosa pauxilla & $\mathrm{T}(\mathrm{T}, \mathrm{N})$ & $D(D, T)$ & $R(R, N)$ \\
\hline Pardosa sp. 1 & $R(R, N)$ & $R(R, N)$ & \\
\hline Pardosa sp. 2 & & $R(R, N)$ & \\
\hline Oxyopes sp. 1 & & $T(D, N)$ & \\
\hline Oxyopes sp. 2 & & $T(R, T)$ & $R(R, N)$ \\
\hline Pisauridae sp. & & $R(R, N)$ & \\
\hline Colonus hesperus & & $R(N, R)$ & $R(R, N)$ \\
\hline Habronattus fallax & $\mathrm{T}(\mathrm{T}, \mathrm{T})$ & $T(T, R)$ & $R(N, T)$ \\
\hline Habronattus nahuatlanus & $T(R, T)$ & $R(R, N)$ & $R(N, R)$ \\
\hline Habronattus mexicanus & $R(N, R)$ & $\mathrm{T}(\mathrm{T}, \mathrm{R})$ & \\
\hline Marpissa pikei & & $R(N, R)$ & \\
\hline Phanias sp. & & & $R(R, N)$ \\
\hline Mexigonus minutus & & $R(R, N)$ & \\
\hline Corythalia sp. & & $R(N, R)$ & $\mathrm{T}(\mathrm{T}, \mathrm{N})$ \\
\hline Sassacus alboguttatus & & $R(R, N)$ & $R(R, N)$ \\
\hline Peckhamia americana & $T(R, T)$ & & \\
\hline Peckhamia sp. & & $R(R, N)$ & \\
\hline Phidippus sp. & & $R(R, N)$ & \\
\hline
\end{tabular}




\begin{tabular}{llll}
\hline Especie & PI & BP & SBC \\
& Td (LL, S) & Td (LL, S) & Td (LL, S) \\
\hline $\begin{array}{l}\text { Metacyrba taeniola taeniola } \\
\text { Selenops sp. }\end{array}$ & & $\mathrm{R}(\mathrm{R}, \mathrm{N})$ & $\mathrm{T}(\mathrm{T}, \mathrm{N})$ \\
\hline $\begin{array}{l}\text { Curicaberis sp. } \\
\text { Xysticus paiutus }\end{array}$ & & $\mathrm{R}(\mathrm{R}, \mathrm{N})$ \\
Xysticus pellax & $\mathrm{T}(\mathrm{T}, \mathrm{N})$ & $\mathrm{T}(\mathrm{T}, \mathrm{N})$ & \\
Meriola decepta & & $\mathrm{R}(\mathrm{R}, \mathrm{N})$ & \\
Trachelas depressus & $\mathrm{T}(\mathrm{R}, \mathrm{R})$ & $\mathrm{R}(\mathrm{R}, \mathrm{N})$ & \\
Zorocrates fuscus & $\mathrm{R}(\mathrm{N}, \mathrm{R})$ & $\mathrm{R}(\mathrm{R}, \mathrm{N})$ & \\
\hline & & $\mathrm{R}(\mathrm{R}, \mathrm{N})$ & $\mathrm{D}(\mathrm{D}, \mathrm{R})$ \\
\hline
\end{tabular}

Para el PI durante el año de recolecta no se encontraron especies dominantes (Fig. 8a), 11 de las especies (55\%) se agruparon en la categoría de especies temporales y las nueve restantes (45\%) fueron especies raras. Durante el periodo de lluvias, $P$. medialis fue la única especie catalogada como dominante, nueve especies (60\%) fueron raras y cinco (33\%) temporales; en la estación seca tres especies (21\%) fueron temporales y 11 (79\%) raras, no se registraron especies dominantes (Cuadro 5).

En el BP considerando todo el año de muestreo (Fig. 8b), las especies $P$. pauxilla y $P$. medialis fueron dominantes (5\%), 24 especies (66\%) se catalogaron como raras y 12 (29\%) fueron temporales. Durante la temporada de lluvias se encontraron 21 especies (66\%) raras, siete (22\%) temporales y las especies dominantes fueron Castianeira azteca Reiskind, 1969, Oxyopes sp. 1, $P$. medialis, y $P$. pauxilla (13\%). Mientras que, en la temporada seca, 11 especies (79\%) fueron raras y tres (21\%) temporales, no se hallaron especies dominantes (Cuadro 5).

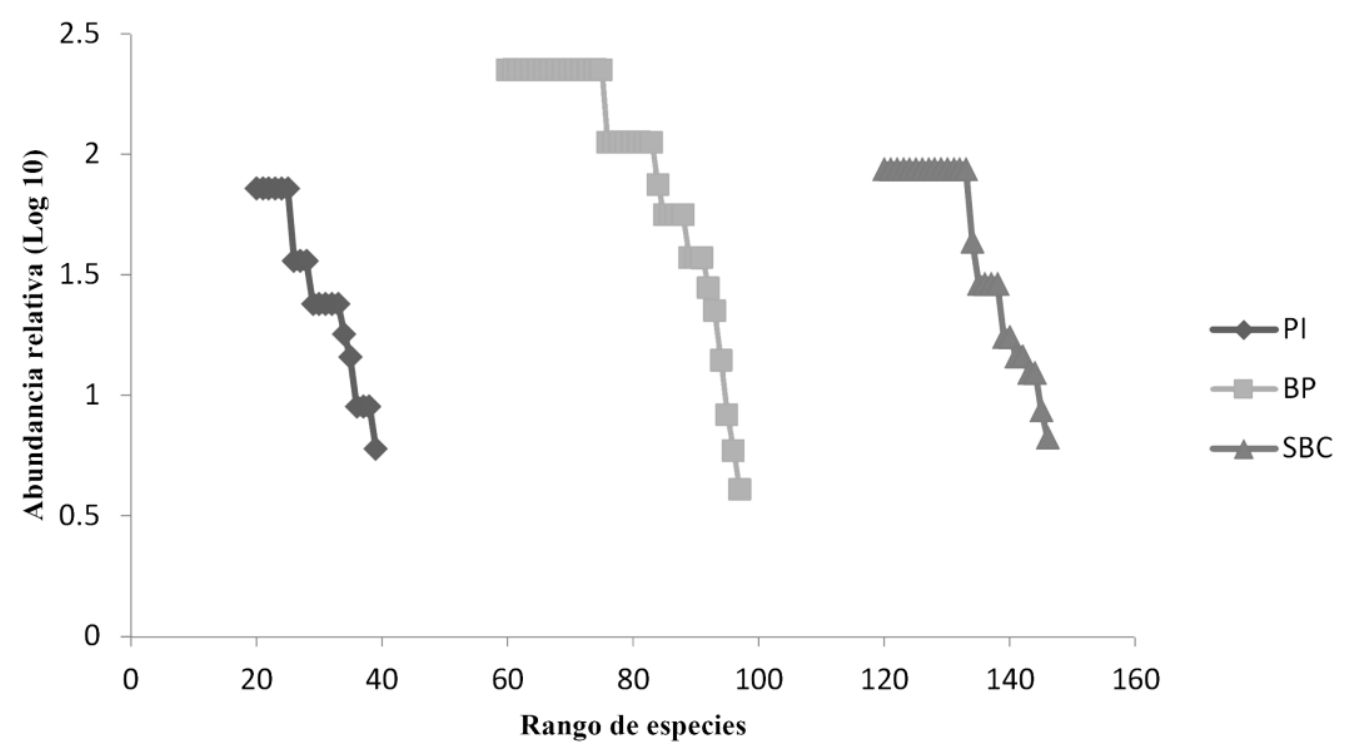


Figura 7. Curvas de rango-abundancia para cada sitio de recolecta. Los valores del eje Y corresponden al logaritmo base 10 de la abundancia relativa. PI: Pastizal Inducido, BP: Bosque de Pino y SBC: Selva Baja Caducifolia.

En la SBC se registró a Zorocrates fuscus Simon, 1888 (4\%) como la única especie dominante durante el año (Fig. 8c), 14 especies (52\%) se catalogaron como raras y 12 (44\%) fueron temporales. En la época de lluvias se encontraron 12 especies (55\%) raras, siete (32\%) temporales y C. azteca, C. lugubris y Z. fuscus (14\%) fueron las especies dominantes; mientras que en la temporada seca no se detectaron especies dominantes, cinco (45\%) fueron raras y seis (55\%) temporales (Cuadro $5)$.
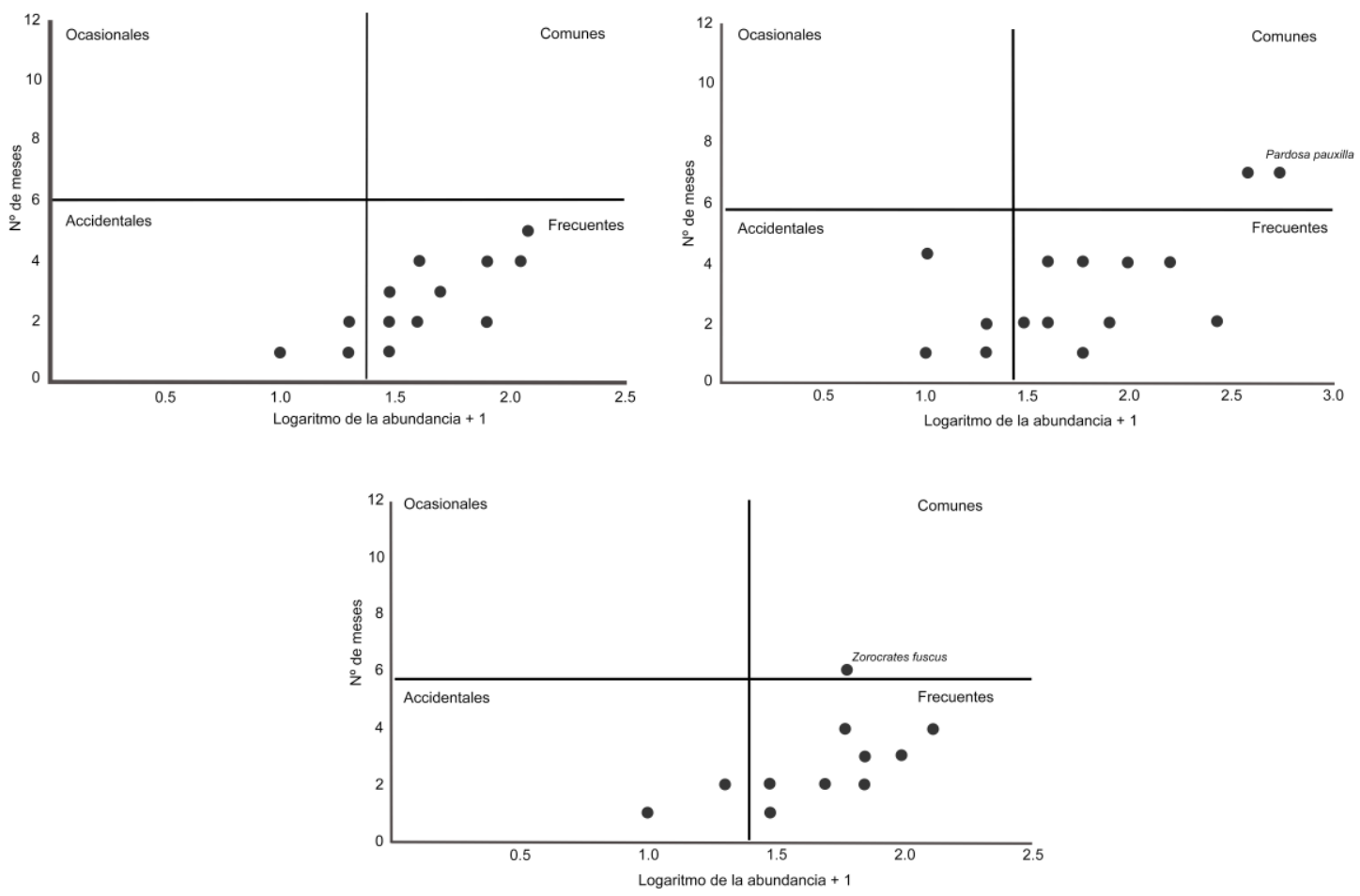

Figura 8. Categorización ecológica de las especies de arañas durante el año. a) Pastizal inducido, b) Bosque de pino y c) Selva baja caducifolia. Para conocer las especies temporales y raras de cada sitio revisar el cuadro 5.

\section{DISCUSIÓN}

Abundancia, riqueza y diversidad. A diferencia de otros estudios que incluyen a otros grupos de arañas, además del RTA y donde existen diferencias estadísticas en la abundancia, riqueza y diversidad entre diversos tipos de vegetación o ecosistemas con distintos grados de perturbación (Campuzano et al., 2016; Maya-Morales et al., 2012; Pinkus et al., 2006, Torres et al., 2017), en esta investigación, las arañas del suelo del clado RTA no presentaron diferencias significativas en los atributos antes indicados y en sitios completamente disimiles, a pesar de que existen antecedentes de que las arañas del suelo proliferan en los espacios libres de vegetación. Estas arañas son capaces de desplazarse en busca de presas (Giraldo et al., 2004), además, pueden soportar condiciones extremas como en las zonas áridas en donde las familias de este grupo presentan las 
abundancias más altas y con mayor riqueza de especies (Russell-Smith, 2002; Bizuet-Flores et al., 2015). Por otra parte, en este trabajo en ninguno de los sitios se encontró diferencia estadística en la abundancia de arañas entre temporadas (lluvias vs secas). Sin embargo, los resultados mostraron un patrón de mayor abundancia de arañas en la temporada de lluvias (Cuadro 2). Resultados similares con arañas del clado RTA fueron reportados por Campuzano y Padilla-Ramírez (2020). También, en otras comunidades de arañas se ha registrado que la mayor abundancia y riqueza de especies de arañas se da en el periodo de lluvias (Llinas-Gutiérrez \& Jiménez, 2004; RodríguezRodríguez et al., 2015). Este patrón puede explicarse al considerar que en tiempo de lluvias hay mayor disponibilidad de recursos derivados del incremento en la cobertura vegetal (Silva \& Coddington 1996; Maya-Morales et al., 2012). Para las arañas del clado RTA, uno de esos recursos podría ser la mayor disponibilidad de presas, tal como lo sugieren Campuzano y Padilla-Ramírez (2020).

En el patrón general, la SBC y el BP tuvieron la mayor riqueza, diversidad (Cuadro 3) y la más alta abundancia de arañas adultas (Cuadro 4). Estos resultados coinciden con lo reportado por Hatley y MacMahon (1980) y Štokmane y Spungis (2016), quienes indicaron que, en sitios con una vegetación estructuralmente más compleja, las abundancias y riqueza de arañas son mayores. Durante la temporada húmeda, en ambos sitios, se encontró una mayor abundancia, riqueza y diversidad a comparación con la temporada seca. La diferencia de estos atributos entre estaciones pudo ser resultado de la influencia del marcado cambio fisonómico entre una época y otra en estos sitios (Vázquez, 1999), pues durante los meses secos hay una considerable pérdida de la estructura vegetal, los estratos herbáceos perecen y en general las plantas inhiben su expansión foliar (Yepes \& Silveira, 2011), además de cambios notorios en la diversidad florística englobada, la disponibilidad de luz, la composición y el volumen de hojarasca, la humedad y la temperatura del suelo (Challenger \& Soberón, 2008).

Integridad de los inventarios. De acuerdo con las categorías propuestas por Cardoso (2009) para determinar qué tan completo es un inventario de artrópodos según el esfuerzo de recolecta, en el periodo de lluvias, se consideró que todos los inventarios son "casi completos o comprensibles" (70-80\%). Durante la temporada de sequía dos son "razonables" (> 50\%) y el inventario del BP se encuentra por debajo de esta categoría (< 50 \%). Para todo el ciclo, dos sitios son "comprensibles" y el de la SBC se encuentra por debajo de la categoría "razonable". Por lo tanto, nuestro método de recolecta fue eficiente en dos de los tres sitios de estudio (Cuadro 4).

Al comparar nuestros resultados con el trabajo de Campuzano y Padilla-Ramírez (2020), en este estudio no se encontraron representantes de las familias Hahniidae y Phrurolitidae. Sin embargo, si se registró a Clubionidae, Oxyopidae, Pisauridae, Selenopidae y Sparassidae, familias que dichos autores no reportaron en su estudio. Lo anterior pude deberse a que el tipo de vegetación y otros factores ambientales limitaron los espacios donde las arañas pueden residir (Corcuera et al., 2010). Asimismo, en el trabajo de Campuzano y Padilla-Ramírez (2020) se evaluó un bosque templado, mientras que en este trabajo se consideraron otros dos tipos de ambientes más. Comparado con otros estudios sistematizados que incluyeron el estrato edáfico y diferentes métodos de recolecta, además de trampas de caída, en el presente trabajo la SBC obtuvo una riqueza de especies del clado RTA similar a la reportada por lbarra et al. (2011) en bosque mesófilo 
de montaña (Chiapas) y Jiménez y Navarrete (2010) en matorral sarcocaule (Baja California Sur), donde se recolectaron 27 y 29 especies de arañas de este clado, respectivamente.

Los hábitos de forrajeo y la estrategia de cacería influyen en el tipo de arañas que pueden capturarse con trampas de caída. El comportamiento sedentario de Selenopidae y Philodromidae explica su baja frecuencia de captura en las trampas (Jiménez \& Navarrete, 2010). Este tipo de método favorece la recolecta de arañas de hábitos cursoriales (Brennan et al., 2005) como Salticidae, Lycosidae, Gnaphosidae y Corinnidae. Se infiere que en el suelo y en estratos no superiores a los $50 \mathrm{~cm}$ las especies de estas familias tienen mejores oportunidades para seleccionar entre un mayor número de hábitats y aprovechar la gran cantidad de microclimas, lo cual permite la persistencia de especies raras (Coddington et al., 1991; Jiménez \& Navarrete, 2010). En comparación con las arañas de redes orbiculares que dependen de la variabilidad estructural y geométrica del sustrato vegetal para poder establecerse (Blanco-Vargas et al., 2003; Romo \& Flores, 2008), razón por la cual presentan mayor diversidad en estrados superiores a los $50 \mathrm{~cm}$ (Höfer \& Brescovit, 2001; Ibarra-Nuñez et al., 2011).

Composición y estructura de la comunidad. La diferencia encontrada en la composición de especies se atribuye al diferente tipo de vegetación presente en cada sitio muestreado. Sin embargo, aunque no se midieron otras variables, se tienen antecedentes de que los cambios en la diversidad florística también modifican la disponibilidad de luz, la composición y el volumen de hojarasca, la humedad y la temperatura del suelo (Uetz, 1975; Trejo, 1999; Challenger \& Soberón, 2008). Todas esas variables influyen sobre las condiciones microclimáticas de los estratos bajos y la superficie del suelo (Mc Caughey et al., 1997, Lindh \& Muir, 2004) determinando la integración de los ensambles de arañas epigeas (Ziesche \& Roth, 2008; Torres et al., 2017).

En cada tipo de vegetación se presentó una estructura de arañas diferente tanto en el ciclo anual (Figs. 8a-c) como temporal (Cuadro 5). Los resultados indicaron que el PI está constituido en su mayoría por especies de Lycosidae y Salticidae (Cuadro 5). Por lo tanto, coincidimos con lo reportado por Escorcia et al. (2012) y Dondale (2017), quienes indicaron que la familia Lycosidae es dominante en sitios abiertos y con baja heterogeneidad vegetal, y con Corcuera et al. (2010), quienes reportaron que la perturbación favorece la presencia y abundancia de especies como $\mathrm{H}$. mexicanus, $H$. fallax y $P$. medialis, especies que se encontraron asociadas al pastizal inducido de La Vega. Por otra parte, Salticidae, Lycosidae y Corinnidae fueron las familias de mayor diversidad en el BP; mientras que la SBC la mayoría de las especies formó parte de Corinnidae además de Gnaphosidae. Las últimas dos familias son habitantes comunes del suelo (Ubick, 2017; Ubick \& Richman, 2017). A saber, la mayoría de las especies de Gnaphosidae tienen grandes áreas de distribución y son más diversas en climas templados donde tienen un amplio rango de preferencias ecológicas, formando comunidades homogéneas que no están fuertemente relacionadas con el tipo de hábitat (Chatzaki et al., 2005). Por su parte, Uetz (1975) registró un aumento en la abundancia de Lycosidae en áreas forestales con estructura de hojarasca poco profunda; mientras que, la abundancia de Gnaphosidae se incrementó en áreas con una cobertura de hojarasca compleja. Este patrón coincidió con nuestros resultados al encontrar mayor cantidad de licósidos en el PI y mayor cantidad de gnafósidos en la SBC. Por otro lado, coincidimos con Corcuera et al. (2010) al encontrar la mayor diversidad de la familia Corinnidae en bosques de 
pino. Tanto Uetz (1975) como Corcuera et al. (2010), sugieren que los patrones de diversidad de las arañas del suelo se deben a la influencia de la hojarasca y de modo parcial a los distintos requerimientos del hábitat que tienen las especies de estas familias.

Los valores del índice de Simpson entre la SBC el BP y el PI tuvieron un rango máximo de diferencia de tres puntos porcentuales, lo que demuestra una alta dominancia entre sitios (Fig. 7). Esto se reflejó en los análisis de categorización ecológica en los cuales la mayoría de las especies se ubicaron como temporales (Cuadro 5), solo P. medialis, P. pauxilla y $Z$. fuscus alcanzaron la categoría de especies dominantes (Fig. 8). Existen antecedentes (Avalos et al., 2007; Bizuet-Flores et al., 2015; Štokmane \& Spungis, 2016) en los cuales también se reporta una estructura de la comunidad de arañas diferente, y lo atribuyen a una alta dominancia entre los sitios que analizan, a la heterogeneidad de los mismos y principalmente a la estructura de la vegetación.

Otra evidencia de la diferencia de la estructura de las comunidades de arañas en este estudio fue que las pocas especies compartidas entre áreas alcanzaron una categoría ecológica distinta dependiendo el sitio y la temporada estacional. Por ejemplo, durante las lluvias $C$. azteca fue dominante en el BP y la SBC, pero no se registró en el $\mathrm{Pl}$; $C$. lugubris fue rara en el $\mathrm{PI}$, temporal en el BP y dominante en la SBC; $P$. pauxilla fue temporal en el PI, dominante en el BP y rara en la $\mathrm{SBC} ; P$. medialis se registró como dominante en el $\mathrm{PI}$ y $\mathrm{BP}$, pero en la $\mathrm{SBC}$ fue rara; mientras que $Z$. fuscus fue rara en el BP y dominante en la SCB (Cuadro 5). En el caso de $Z$. fuscus hay antecedentes de que es más abundante en sitos con PI (Desales-Lara, 2010) y sitios antropogénicos (Desales-Lara et al., 2013). Esos resultados contrastan con el presente trabajo, ya que dicha especie en este estudio fue dominante en el sitio con SBC (Cuadro 5; Fig. 8c). Sin embargo, se coincide con lo reportado por Campuzano y Padilla-Ramírez (2020) al encontrar a $Z$. fuscus con abundancias altas en temporada de lluvias.

\section{CONCLUSIONES}

En suma, las comunidades de arañas del clado RTA presentan cambios en la composición y estructura entre sitios disimiles en la vegetación. Además, son sensibles a los cambios estacionales, pues se muestra una tendencia de mayor riqueza y diversidad de las poblaciones en la temporada húmeda del año. Aunque este trabajo no evaluó de manera directa la influencia de variables microambientales, es posible que factores asociados con la complejidad de la hojarasca, la presencia de competidores, la disponibilidad de espacios intersticiales para construir refugios, la cantidad de presas y variaciones microclimáticas, jueguen un papel importante en el ensamble de las comunidades. Para establecer dichas asociaciones y tener un mejor entendimiento en la estructura de comunidades de arañas, futuros trabajos deberán enfocarse en el análisis de la hojarasca como factor determinante en el ensamble de comunidades de arañas RTA. Conjuntamente, sería conveniente evaluar por separado el efecto de las temporadas sobre los distintos gremios de arañas. Además, es recomendable incrementar el esfuerzo de muestreo en los meses secos del año, ya sea aumentado el área de muestreo o adicionando otro método de recolecta como búsqueda directa o cernido de hojarasca para asegurar la inclusión de las arañas RTA no corredoras. 
Agradecimientos. A Jorge Padilla Ramírez por haber financiado el estudio, Petra Sánchez-Nava por permitirnos hacer uso de las instalaciones del Centro de Investigación de Recursos Bióticos (CIRB) de la UAEMex. A Griselda Montiel-Parra por permitirnos trabajar en el laboratorio de la CNAC. A Antonio Ayala por conseguir los permisos para muestrear en los sitios. Asimismo, deseamos agradecer a Luis A. Matías por su colaboración durante el trabajo de campo, Laura Olguín por la ayuda prestada para la determinación de las arañas lobo. Finalmente, agradecemos a Gabriel Gutiérrez, Ángel Rodríguez, Margarita Ojeda, Guillermo Ibarra Núñez y a los revisores anónimos por los valiosos comentarios hechos al manuscrito.

\section{LITERATURA CITADA}

Avalos, G., Rubio, G. D., Bar, M. E., González, A. (2007) Arañas (Arachnida: Araneae) asociadas a dos bosques degradados del Chaco húmedo en Corrientes, Argentina. Revista de Biología Tropical, 55, 899-909. Disponible en: https://www.redalyc.org/pdf/449/44912352015.pdf (Consultado 03 octubre 2019).

Bizuet-Flores, M. Y., Jiménez-Jiménez, M. L., Zavala-Hurtado, A., Corcuera, P. (2015) Diversity patterns of ground dwelling spiders (Arachnida: Araneae) in five prevailing plant communities of the Cuatro Ciénegas Basin, Coahuila, Mexico. Revista Mexicana de Biodiversidad, 86, 153-163.

Blanco-Vargas, E., Amat, G., Flórez, E. (2003) Araneofauna orbitelar (Araneae: Orbiculariae) de los Andes de Colombia: comunidades en hábitats bajo regeneración. Revista Ibérica de Aracnología, 7, 189-203. Disponible en: http://sea-entomologia.org/PDF/RIA_7/R07-026189.pdf (Consultado 03 octubre 2019).

Brennan, K., Majer, J., Moir, M. (2005) Refining sampling protocols for inventorying invertebrate biodiversity: influence of drift-fence length and pitfall trap diameter on spiders. The Journal of Arachnology, 33, 681-702. Disponible en: https://www.jstor.org/stable/4129870 (Consultado 12 abril 2021).

Campuzano, E., Ibarra-Núñez, G., Chamé-Vázquez, E., Montaño-Moreno, H. (2016) Understory spider assemblages from a cloud forest in Chiapas, Mexico, and their relationships to environmental variables. Arthropod-Plant Interactions, 10, 237-248. https://doi.org/10.1007/s11829-016-9426-z

Campuzano, E., Ibarra-Núñez, G., Gómez, J., Angulo, G. (2019) Spiders (Arachnida: Araneae) of the tropical mountain cloud forest from El Triunfo Biosphere Reserve, Mexico. Acta Zoológica Mexicana (nueva serie), 35, 1-19. https://doi.org/10.21829/azm.2019.3502092

Campuzano, E., Ibarra-Núñez, G., Machkour-M'Rabet, S., Morón-Ríos, A., Jiménez, M. L. (2020) Diversity and seasonal variation of ground and understory spiders from a tropical mountain cloud forest. Insect Science, 27, 4, 826-844. https://doi.org/10.1111/1744-7917.12693

Campuzano, E., Padilla-Ramírez, J. (2020) Spatial and seasonal patterns of ground-dwelling spider assemblages belonging to the retrolateral tibial apophysis clade (Araneae: Araneomorphae) in two remnants of pine-oak forest from Mexico. Studies on Neotropical Fauna and Environment, 1-14. https://doi.org/10.1080/01650521.2020.1806008 
Cardoso, P. (2009) Standardization and optimization of arthropod inventories- the case of Iberian spiders. Biodiversity and Conservation, 18, 3949-3962. https://doi.org/10.1007/s10531-009-9690-7

Challenger, A., Soberón, J. (2008) Los ecosistemas terrestres. Pp. 87-108. En: J. Soberón, G. Halffter y J. Llorente-Bousquets, Capital Natural de México Volúmen I. CONABIO, México, Ciudad de México.

Chatzaki, M., Lymberakis, P., Markakis, G., Mylonas, M. (2005) The distribution of ground spiders (Araneae, Gnaphosidae) along the altitudinal gradient of Crete, Greece: species richness, activity and altitudinal range. Journal of Biogeography, 32, 813-331.

https://doi.org/10.1111/j.1365-2699.2004.01189.x

Cipriano-Anastasio, J., Cruz-Salazar, F. L., López-Mancilla, A., Hernández-Hernández, J., Lara-Hernández, J. L., Cruz-Monterrubio, A. L. (2019) Estudio preliminar de la arácnofauna en la ribera del río Chinguiñoso, Huejutla de Reyes, Hidalgo, México. Revista Biológico Agropecuaria Tuxpan, 7 (2), 105-113.

https://doi.org/10.47808/revistabioagro.v7i2.54

Coddington, J. A., Griswold, C. E., Silva, D., Peñaranda, E., Larcher, S. F. (1991) Designing and Testing Sampling Protocols to Estimate Biodiversity in Tropical Ecosystems. Pp. 44-60. In: E. C. Dudley (Ed.). The Unity of Evolutionary Biology: Proceedings of the Fourth International Congress of Systematic and Evolutionary Biology, 1. Dioscorides Press, Portland.

Colwell, R. K. (2013) EstimateS: statistical estimation of species richness and shared species from samples. Version 9.1.0. Disponible en: http://viceroy.eeb.uconn.edu/estimates (Consultado 17 marzo 2016).

Colwell, R. K., Coddington, J. A. (1994). Estimating terrestrial biodiversity through extrapolation. Philosophical Transactions of the Royal Society of London Series B-Biological Sciences, 345, 101-118.

https://doi.org/10.1098/rstb.1994.0091

Contreras, M., Agramont, A., Regil, H., Franco, S. (2010) Superficie forestal. Pp. 15-58. In: G. Nava, A. Agramont, H. Regil, C. Velázquez, C. Arriaga, S. Franco (Eds.). Los bosques y selvas del Estado de México. Universidad Autónoma del Estado de México UAEMéx, Estado de México, México.

Corcuera, P., Jiménez, M. L., Valverde, P. (2008) Does the microarchitecture of Mexican dry forest foliage influence spider distribution? Journal of Arachnology, 36, 552-556. https://doi.org/10.1636/t05-20.1

Corcuera, P., Valverde, P., Zavala-Hurtado, J., De la Rosa, G., Durán, C. (2010) Non weaving spiders on native woodlands and Eucalyptus plantations in Western Mexico: diversity and distribution patterns. Journal of Insect Conservation, 14, 711-719.

https://doi.org/10.1007/s10841-010-9301-7

Cruz-Pérez, A., Pérez-De la Cruz, M. Sánchez-Soto, S., Torres-De la Cruz, M. (2015) Fluctuación poblacional de arañas (Araneae: Tetragnathidae, Pholcidae) en el agroecosistema de cacao en Tabasco, México. Revista Colombiana de entomología, 41 (1), 132-138. Disponible en: http://www.scielo.org.co/pdf/rcen/v41n1/v41n1a20.pdf (Consultado 12 abril 2021).

Desales-Lara, M. A. (2010) Contribución a la historia natural de Zorocrates fuscus Simon, 1888 (Araneae: Zorocratidae). Entomología Mexicana, 9, 39-43. 
Desales-Lara, M. A. (2012) Descripción de una nueva especie del género Fillistatinella Gertsch \& Ivie, 1936 (Araneae: Filistatidae) de Toluca, México. Revista Ibérica de Aracnología, 21, 5155.

Desales-Lara, M. A. (2014) Araneofauna (Arachnida: Araneae) del Estado de México, México. Acta Zoológica Mexicana (nueva serie), 30 (2), 298-320. https://doi.org/10.21829/azm.2014.302104

Desales-Lara, M. A., Francke, O., Sánchez-Nava, P. (2013) Diversidad de arañas (Arachnida: Araneae) en hábitats antropogénicos. Revista Mexicana de Biodiversidad, 84, 291-305. https://doi.org/10.7550/rmb.31708

Dondale, D. (2017) Lycosidae. Pp. 178-182. In: D. Ubick, P. Paquin, P. E. Cushing, V. Roth (Eds.). Spiders of North America: an identification manual. American Arachnological Society, United States of America, Keene, New Hampshire.

Escorcia, R., Martínez, N., Silva, J. (2012) Estudio de la diversidad de arañas de un bosque seco tropical (BS-T) en Sabanalarga, Atlántico, Colombia. Boletín Científico Museo de Historia Natural, Universidad de Caldas, 16 (1), 247-260. Disponible en: http://www.scielo.org.co/scielo.php?pid=S012330682012000100021\&script=sci_abstract\&tlng=es (Consultado 12 abril 2021).

Foelix, R. (2011) Biology of Spiders, $3^{a}$. Oxford University Press. United States of America, 338 pp. Garrison, N., Rodriguez, J., Agnarsson, I., Coddington, J., Griswold, C., Hamilton, C., Hedin, M., Kocot, K., Ledford, J., Bond, J. (2016) Spider phylogenomics: untangling the spider tree of life. PeerJ, 4:e1719, 1-35. https://doi.org/10.7717/peerj.1719

Giraldo, A., Pérez, D., Arellano, G. (2004) Respuesta de la comunidad de arañas epigeas (Araneae) en las Lomas de Lachay, Perú, ante la ocurrencia del evento del niño 1997-98. Ecología aplicada, 3 (1, 2), 45-58. Disponible en: https://www.redalyc.org/pdf/341/34130207.pdf (Consultado 12 abril 2021).

Griswold, C., Ramírez, M. (2017) Phylogeny of spiders. Pp. 17-29. In: D. Ubick, P. Paquin, P. E. Cushing, V. Roth (Eds.). Spiders of North America: an identification manual. American Arachnological Society, United States of America, Keene, New Hampshire.

Guerrero-Fuentes, D., Desales-Lara, M. (2020) Nuevos registros de arañas (Araneae) del Estado de México, México. Revista Ibérica de Aracnología, 36, 147-149. Disponible en: http://seaentomologia.org/gia/ria_36_147_149.html (Consultado 12 abril 2021).

Guerrero-Fuentes, D., Francke, O. (2019) Taxonomic revision of Anicius Chamberlin, 1925 (Araneae: Salticidae), with five new species of jumping spiders from Mexico. Zootaxa, 4638 (4), 485-506. https://doi.org/10.11646/zootaxa.4638.4.2

Hammer, Ø., Harper, D. A. T., Ryan, P. D. (2001) PAST: paleontological statistics software package for education and data analysis. Palaeontol Electron, 4, 1-9.

Hatley, C., Macmahon, J. (1980). Spider community organization: seasonal variation and the role of vegetation architecture. Environmental Entomology, 9, 632-639. Disponible en: https://core.ac.uk/download/pdf/19685626.pdf (Consultado 12 abril 2021).

Höfer, H., Brescovit, A. D. (2001) Species and guild structure of a Neotropical spider assemblage (Araneae; Reserva Ducke, Amazonas, Brazil). Andrias, 15, 99-120. Disponible en: https://www.zobodat.at/pdf/Andrias_15_0099-0119.pdf (Consultado 12 abril 2021). 
Hoffmann, A. (1976) Relación bibliográfica preliminar de las arañas de México (Arachnida: Araneae). Publicaciones especiales No. 3. Instituto de Biología, UNAM, México, 117 pp.

Ibarra-Núñez, G. (1990) Los artrópodos asociados a cafetos en un cafetal mixto del Soconusco, Chiapas, México, I. Variedad y abundancia. Folia Entomológica Mexicana, 79, 207-231. Disponible en: https://www.researchgate.net/publication/285770570 (Consultado 12 abril 2021).

Ibarra-Núñez, G., García-Ballinas, J. (1998) Diversidad de tres familias de arañas tejedoras (Araneae: Araneidae, Tetragnathidae, Theridiidae) en cafetales del Soconusco, Chiapas, México. Folia Entomológica Mexicana, 102, 11-20.

Ibarra-Núñez, G., Maya-Morales, J., Chamé-Vázquez, D. (2011) Las arañas del bosque mesófilo de montaña de la Reserva de la Biosfera Volcán Tacaná, Chiapas, México. Revista Mexicana de Biodiversidad, 82, 1183-1193. http://dx.doi.org/10.22201/ib.20078706e.2011.4.736

INEGI, Instituto Nacional de Estadística y Geografía (2009) Prontuario de información geográfica municipal de los Estados Unidos Mexicanos, Ecatepec de Morelos, México. INEGI, 9 pp.

James, F. C., Rathbun, S. (1981) Rarefaction, relative abundance, and diversity of avian communities. The Auk, 98, 785-800. Disponible en: https://www.jstor.org/stable/4085899 (Consultado 12 abril 2021).

Jiménez, M. L., Palacios-Cardiel, C., Chávez-Lara, I. (2020) Las Arañas (Arachnida: Araneae) de Los Médanos de Samalayuca, Chihuahua y nuevos registros de especies para México. Acta Zoológica Mexicana (nueva serie), 36, 1-23. https://doi.org/10.21829/azm.2020.3612205

Jiménez, M. L., Navarrete, J. (2010) Fauna de arañas del suelo de una comunidad árida-tropical en Baja California Sur, México. Revista Mexicana de Biodiversidad, 81, 417-426.

Jost, L. (2006) Entropy and diversity. Oikos, 113 (2), 363-375. Disponible en: https://www.jstor.org/stable/40234813 (Consultado 12 abril 2021).

Lindh, B., Muir, P. (2004) Understory vegetation in young Douglas-fir forests: does thinning help restore old-growth composition? Forest Ecology and Management, 192, 285-296. https://doi.org/10.1016/j.foreco.2004.01.018

Llinas-Gutiérrez, J., Jiménez, M. L. (2004) Arañas de humedales del sur de Baja California, México. Anales del Instituto de Biología, UNAM, Serie Zooloógica, 75 (2), 283-302. Disponible en: http://www.revistas.unam.mx/index.php/zoo/article/view/14664 (Consultado 12 abril 2021).

López-Mejía, M., Moreno, C., Zuria, I., Sánchez-Rojas, G., Rojas-Martínez, A. (2017) Comparación de dos métodos para analizar la proporción de riqueza de especies entre comunidades: un ejemplo con murciélagos de selvas y hábitats modificados. Revista Mexicana de Biodiversidad, 88, 183-191.

http://dx.doi.org/10.1016/j.rmb.2017.01.008

Lucio-Palacio, C., Ibarra-Núñez, G. (2015) Arañas arborícolas de cacaotales con diferente tipo de manejo en Chiapas, México. Revista Mexicana de Biodiversidad, 86, 143-152. https://doi.org/10.7550/rmb.41027

Maldonado-Carrizales, J., Ponce-Saavedra, J. (2017) Arañas Saltarinas (Araneae: Salticidae) en dos sitios contrastantes en grado de antropización en Morelia Michoacán, México. 
Entomología Mexicana, 4, 597-603. Disponible en: http://www.entomologia.socmexent.org/revista/2017/EUL/EM2692017_597-603.pdf (Consultado 12 abril 2021).

Maldonado-Carrizales, J., Quijano-Ravell, A., Guzmán-García, C., Ponce-Saavedra, J. (2018) Arañas (Araneae: Araneomorphae) antrópicas de Morelia, Michoacán, México. Entomología Mexicana, 22-28. 5, Disponible en: http://www.entomologia.socmexent.org/revista/2018/AA/AA\%20022-028.pdf (Consultado 12 abril 2021).

Marín, L., Philpott, S., De la Mora, A., Ibarra-Núñez, G., Tryban, S., Perfecto, I. (2016) Response of ground spiders and landscape factors in a Mexican coffee landscape. Agriculture, Ecosystems and Environment, 222, 80-92. https://doi.org/10.1016/j.agee.2016.01.051

Marmolejo, A. M. (2010) Manifestación de impacto ambiental, modalidad regional, sector hidráulico, presa de almacenamiento "EL ARENAL" Ixtapan de la Sal y Tonatico, México. Centro de estudios territoriales aplicados, Facultad de Planeación Urbana y Regional, Universidad Autónoma del Estado de México, México, 375 pp.

Márquez, M. J. (2004) Probabilidad y estadística para ciencias químico biológicas. Facultad de Estudios Superiores Zaragoza, Universidad Nacional Autónoma de México, México, CDMX, $626 \mathrm{pp}$.

Maya-Morales, J., Ibarra-Núñez, G., León-Cortés, J., Infante, F. (2012) Understory spider diversity in two remnants of tropical montane cloud forest in Chiapas, Mexico. Journal of Insect Conservation, 16, 25-38.

https://doi.org/10.1007/s10841-011-9391-x

Mc Caughey, J., Amiro, B., Robertson, A., Spittlehouse, D. (1997) Forest environments. Pp. 247276. In: W. G. Bailey, T. R. Oke, W. R. Rouse (Eds.). The Surface Climates of Canada. McGill Queen's University Press, Montreal.

Moreno, C. (2001) Métodos para medir la biodiversidad. M\&T-Manuales y Tesis Vol. 1. Sociedad Entomológica Aragonesa. Zaragoza, España, 84 pp.

Moreno, C., Barragán, F., Pineda, E., Pavón, N. (2011) Reanálisis de la diversidad alfa: alternativas para interpretar y comparar información sobre comunidades ecológicas. Revista Mexicana de Biodiversidad, 82, 1249-1261. Disponible en: https://www.redalyc.org/articulo.oa?id=42520885019 (Consultado 12 abril 2021).

Pinkus, M., Ibarra-Núñez, G., Parra-Tabla, V., García-Ballinas, J., Hénaut, Y. (2006) Spider diversity in coffee plantations with different management in Southeast Mexico. The Journal of Arachnology, 34, 104-112. https://doi.org/10.1636/M03-044.1

Platnick, N. I. (2020) Spiders of the World. A natural history. Princeton University Press. USA, 256 pp.

Platnick, N., Shadab, M. (1975) A revision of the spider genus Gnaphosa (Araneae: Gnaphosidae). Bulletin of the American Museum of Natural History, 155, 1-66.

Platnick, N., Shadab, M. (1977) A revision of the spider genera Herpyllus and Scotophaeus (Araneae: Gnaphosidae). Bulletin of the American Museum of Natural History, 159, 1-44.

Platnick, N., Shadab, M. (1980) A revision of the spider genus Cesonia (Araneae: Gnaphosidae). Bulletin of the American Museum of Natural History, 165, 337-385. 
Platnick, N., Shadab, M. (1982) A revisión of the spider genus Drasyllus (Araneae: Gnaphosidae). Bulletin of the American Museum of Natural History, 173, 1-97.

Ramírez, M. J. (2014) The morphology and phylogeny of dionychan spiders (Araneae: Araneomorphae). Bulletin of the American Museum of Natural History, 1, 1-374. https://doi.org/10.1206/821.1

Rivera-Quiroz, F., Garcilazo-Cruz, U., Álvarez-Padilla, F. (2016) Spider cyberdiversity (Araneae: Araneomorphae) in an ecoturistic tropical forest fragment in Xilitla, Mexico. Revista Mexicana de Biodiversidad, 87, 1023-1032. http://dx.doi.org/10.1016/j.rmb.2016.07.011

Rodríguez-Rodríguez, S., Solís-Catalán, K., Valdez-Mondragón, A. (2015) Diversity and seasonal abundance of anthropogenic spiders (Arachnida: Araneae) in different urban zones of the city of Chilpancingo, Guerrero, Mexico. Revista Mexicana de Biodiversidad, 86, 962-971.

https://doi.org/10.1016/j.rmb.2015.09.002

Romo, M. T., Flores, E. (2008) Comunidad de arañas orbitelares (Araneae: Orbiculariae), asociada al bosque alto andino del santuario flora y fauna galeras, Nariño, Colombia. Boletín científico del museo de historia natural, 13 (1), 114-126. Disponible en: http://www.scielo.org.co/scielo.php?script=sci_arttext\&pid=S0123-30682009000100010 (Consultado 12 abril 2021).

Roskov, Y., Ower, G., Orrell, T., Nicolson, D., Bailly, N., Kirk, P. M., Bourgoin, T., DeWalt, R. E., Decock, W., Nieukerken, E. van, Zarucchi, J., Penev, L. (2019) Species 2000 \& ITIS Catalogue of Life, 29th January 2019. Species 2000: Naturalis, Leiden, The Netherlands. Disponible en: http//www.catalogueoflife.org (Consultado 10 enero 2020).

Russell-Smith, A. (2002) A comparison of the diversity and composition of the ground-active spider in Mkomazi Game Reserve, Tanzania and Etosha National Park, Namibia. Journal of Arachnology, 30, 383-388. Disponible en: https://www.jstor.org/stable/3706282 (Consultado 12 abril 2021).

Salazar-Olivo, C., Solís-Rojas, C. (2015) Araneofauna urbana (Arachnida: Araneae) de Ciudad Victoria, Tamaulipas, México. Acta Zoológica Mexicana (nueva serie), 31 (1), 55-66. https://doi.org/10.21829/azm.2015.311505

Silva, D., Coddington, J. (1996) The biodiversity of Pakitza (Madre de Dios, Perú): species richness and notes on community structure. Pp. 253-311. In: D. Wilson, A. Sandoval (Eds.). The Biodiversity of Southeastern Perú. Smithsonian Institution, United States of America, Washington D.C.

Sokal, R., Rohlf, F. (1981) Biometry. W. H. Freeman Inc. Estados Unidos, 859 pp.

Štokmane, M., Spungis, V. (2016) The influence of vegetation structure on spider species richness, diversity and community organization in the Apšuciems calcareous fen, Latvia. Animal Biodiversity and Conservation, 39.2, 221-236. Disponible en: http://abc.museucienciesjournals.cat/files/ABC_39-2_pp_221-236-color.pdf (Consultado 12 abril 2021).

Torres, V., González-Reyes, A., Corronca, J. (2017) Diversidad taxonómica y funcional de arañas (Araneae) epigeas en bosques nativos de las Yungas (Salta, Argentina). Caldasia, 39 (2), 326-344.

https://doi.org/10.15446/caldasia.v39n2.63071 
Trejo, I. (1999) El clima de la selva baja caducifolia en México. Investigaciones geográficas, 39, 4052.

Ubick, D. (2017) Gnaphosidae. Pp. 106-111. In: D. Ubick, P. Paquin, P. E. Cushing, V. Roth (Eds.). Spiders of North America: an identification manual. American Arachnological Society, United States of America, Keene, New Hampshire.

Ubick, D., Richman, D. (2017) Corinnidae. Pp. 79-82. In: D. Ubick, P. Paquin, P. E. Cushing, V. Roth (Eds.). Spiders of North America: an identification manual. American Arachnological Society, United States of America, Keene, New Hampshire.

Ubick, D., Paquin, P., Cushing, P. E., Roth, V. (2017) Spiders of North America: an identification manual. American Arachnological Society, USA, Keene, New Hampshire, 376 pp.

Uetz, G. (1975) Temporal and spatial variation in species diversity of wandering spiders (Araneae) in deciduous forest litter. Environmental Entomology, 4 (5), 719-724. https://doi.org/10.1093/ee/4.5.719

Vázquez, O. (1999) Tonatico, Monografía Municipal. Instituto Mexiquense de Cultura, México, 183 pp.

Wheeler, C., Coddington, J., Crowley, L., Dimitrov, D., Goloboff, P., Griswold, C., Hormiga, G., Prendini, L., Ramírez, M., Sierwald, P., Almeida-Silva, L., Alvarez-Padilla, F., Arnedo, M., Benavides, L., Benjamin, S., Bond, J., Grismado, C., Hasan, E., Hedin, M., Izquierdo, M., Labarque, F., Ledford, J., Lopardo, L., Maddison, W., Miller, J., Piacentinni, L., Platnick, N., Polotow, D., Silva-Dávila, D., Scharff, N., Szüts, T., Ubick, D., Vink, C., Wood, H., Zhanng, J. (2016) The spider tree of life: phylogeny of Araneae based on target-gene analyses from an extensive taxon sampling. Cladistics, 1-4. https://doi.org/10.1111/cla.12182

WSC (2020) World Spider Catalog, Ver. 21.5, Natural History Museum Bern. Disponible en: https://wsc.nmbe.ch (Consultado 25 junio 2020).

Yepes, A., Silveira, M. (2011) Respuestas de las plantas ante los factores ambientales del cambio climático global (revisión). Colombia Forestal, 14 (2), 213-232. Disponible en: https://www.redalyc.org/pdf/4239/423939616005.pdf (Consultado 12 abril 2021).

Ziesche, T., Roth, M. (2008) Influence of environmental parameters on small-scale distribution of soil-dwelling spiders in forests: What makes the difference, tree species or microhabitat? Forest Ecology and Management, 255 (3), 738-752.

https://doi.org/10.1016/j.foreco.2007.09.060 